\title{
Tokamak-edge toroidal rotation due to inhomogeneous transport and geodesic curvature
}

\author{
T. Stoltzfus-Dueck ${ }^{a)}$ \\ Max-Planck-Institut für Plasmaphysik, EURATOM Association, Boltzmannstraße 2, 85748 Garching, \\ Germany
}

(Dated: June 4, 2012)

In a model kinetic ion transport equation for the pedestal and scrape-off layer, passing-ion drift orbit excursions interact with spatially-inhomogeneous but purely diffusive transport to cause the orbit-averaged diffusivities to depend on the sign of $v_{\|}$, preferentially transporting counter-current ions for realistic parameter values. The resulting pedestal-top intrinsic rotation is typically co-current, reaches experimentally relevant values, and is proportional to pedestal-top ion temperature $\left.T_{i}\right|_{\mathrm{pt}}$ over plasma current $I_{p}$, as observed in experiment. The rotation drive is independent of the toroidal velocity and its radial gradient, representing a residual stress. Co-current spin-up at the L-H transition is expected due to increasing $\left.T_{i}\right|_{\text {pt }}$ and a steepening of the turbulence intensity gradient. A more inboard (outboard) X-point leads to additional co- (counter-) current rotation drive. Beyond intrinsic rotation, comparison of heat and momentum transport reveals that neutral beam injection must be significantly unbalanced in the counter-current direction to cause zero toroidal rotation at the pedestal top.

\section{INTRODUCTION}

Toroidal rotation plays an important role in tokamak performance, stabilizing resistive wall modes $^{1}$ and contributing to $E_{r}$ shear, believed to suppress turbulent transport. ${ }^{2}$ While neutral beam (NBI) heating applies significant torque to present-day tokamaks, future burning plasma experiments like ITER will receive relatively little external torque. ${ }^{3}$ The experimental observation of intrinsic rotation in the absence of applied torque ${ }^{4-15}$ is therefore of particular interest. In the edge, this intrinsic rotation is essentially always directed with the plasma current (co-current). ${ }^{4-10}$ The effect can be of large magnitude, leading to pedestal-top intrinsic rotation velocities reaching tenths of the local ion thermal speed $\left.v_{t i}\right|_{\mathrm{pt}} \doteq{\sqrt{\left.T_{i}\right|_{\mathrm{pt}} / m_{i}}}^{7-10}$ and intrinsic torque comparable with the torque applied by a neutral beam source. ${ }^{16}$ Although nontrivial core rotation profiles are often observed, the edge region appears to play a significant, sometimes dominant, role. ${ }^{5-14}$ Dedicated experiments are beginning to uncover local parameter scalings in the edge, where it appears that the rotation speed may be proportional to $T_{i}$ or its gradient. ${ }^{10,15}$

A broad spectrum of theoretical models have been put forward to explain the rotation observations. Neoclassical models ${ }^{17-23}$ have matched some experimental features, ${ }^{12,22,24}$ but predict toroidal viscosities far lower than observed in experiment. ${ }^{6,12-14,24-27}$ Turbulent models have primarily focused on core physics, dominantly using quasilinear approximations, ${ }^{28-32}$ mostly based on ITG $^{33-40}$ and trapped electron ${ }^{39-41}$ modes, identifying momentum pinches due to radial electric field $\left(E_{r}\right)$ shear, ${ }^{38,39}$ temperature gradients, ${ }^{28,32}$ and magnetic inhomogeneity, ${ }^{32-36,41}$ as well as residual stress due to

\footnotetext{
a) Electronic mail: tstoltzf@ipp.mpg.de
}

the ion pressure gradient, ${ }^{29}$ up-down asymmetric magnetic geometry, ${ }^{40}$ the polarization drift, ${ }^{30,37}$ and Alfvén waves. ${ }^{31}$ Effects due to non-resonant turbulent parallel acceleration of ions have also been estimated. ${ }^{42-44}$ Stringer spin-up-type models ${ }^{45,46}$ have been applied to toroidal rotation problems, ${ }^{47-49}$ although the resulting toroidal rotation drive is much weaker than the poloidal drive. ${ }^{48}$ A number of scrape-off-layer (SOL) effects have been put forward, such as nonvanishing radial current, ${ }^{50,51}$ ion orbit losses, ${ }^{10}$ and inward diffusion of transport-driven SOL parallel flows, ${ }^{52,53}$ but without self-consistent consideration of the confined plasma. Nonlinear turbulent simulations have exhibited nondiffusive toroidal momentum transport, ${ }^{54}$ interpreted as resulting from the $\mathbf{E} \times \mathbf{B}$ and Coriolis pinches ${ }^{55}$ or from residual stress due to zonal flow shear or a turbulence intensity gradient, ${ }^{56-58}$ examined effects of rotation on internal transport barriers, ${ }^{59}$ and demonstrated the reduction of quasilinear momentum transport effects by damped modes. ${ }^{60}$ It has been argued that additional terms must be introduced to gyrokinetic formulations to accurately treat the momentum transport when toroidal rotation is small, ${ }^{61}$ while a general toroidal momentum conservation theorem covering most present-day formulations has been rigorously proved. ${ }^{62,63}$

Turbulence and transport in the tokamak edge involves orderings that differ significantly from the core, due principally to the extremely steep edge gradients of plasma parameters. ${ }^{64,65}$ In the edge of present-day tokamaks, equilibrium plasma densities and temperatures vary rapidly with minor-radial position $r$ on a length scale $L_{\perp}$ around a centimeter or a few,${ }^{66-68}$ much shorter than the characteristic parallel length scale of turbulent fluctuations $k_{\|}^{-1} \sim q R_{0}$ (with $q$ the safety factor and $R_{0}$ the major radius), several to many meters. ${ }^{69,70}$ The significant ion thermal Mach numbers observed at the pedestal top ${ }^{7-10}$ imply that minor-radial variation of the toroidal rotation velocity can achieve similar steepness. 
The steep density and temperature gradients drive fluctuations into the strong turbulence regime, with statistics widely differing from the linear estimates used in quasilinear theory. ${ }^{64,71-74}$ The extreme anisotropy $k_{\|} L_{\perp} \lll 1$ implies that turbulent parallel acceleration of ions results in relatively weak nondiffusive momentum transport in the edge. For example, following Ref. 42, one may compare a simple momentum diffusion term $\propto D_{r, r}$ with potential intrinsic-rotation-driving cross-terms $\propto$ $D_{r, v_{\|}}, D_{v_{\|}, r}$, finding the latter to scale relative to the former as $k_{\|} L_{\perp} / k_{\perp} \rho_{s}$, with $k_{\perp}$ a typical perpendicular wave number of the fluctuations, $\rho_{s} \doteq c_{s} / \Omega_{i}, c_{s} \doteq\left(T_{e} / m_{i}\right)^{1 / 2}$, and $\Omega_{i}$ the ion cyclotron frequency. Much smaller than one for typical edge parameters, this ratio exhibits the basic scaling of mechanisms relying on turbulent parallel acceleration, ${ }^{75}$ which are generally further reduced by the fact that the required symmetry-breaking $\left\langle k_{\|}\right\rangle$is much smaller than the rms $k_{\|}$used in the present estimate. ${ }^{30,76}$ Mechanisms dependent on magnetic inhomogeneity bring in Mach number variations on the $R_{0}$ scale,$^{32-35,40,41}$ also much too gradual to explain the edge rotation gradients on the $L_{\perp}$ scale.

Situated on the open-closed field line boundary, the edge and SOL physics are inherently nonlocal. For example, particle and energy balance implies that the ion thermal transit time and turbulent diffusion time must be comparable in the SOL. ${ }^{77}$ Since plasma parameters and gradients are continuous over the LCFS, ${ }^{66,67}$ this ordering also holds in the outer edge, ${ }^{78}$ fundamentally violating the slow-transport ordering underlying neoclassical theory ${ }^{79,80}$ and other radially-local models. ${ }^{61}$ The unnormalized amplitude of potential fluctuations $\tilde{\phi}$ also varies rapidly, decreasing with increasing $r$ on a short length scale $L_{\phi} \sim L_{\perp}$, with $L_{\phi}$ typically between one and several times $L_{T e}$, the decay length for electron temperature $T_{e} \cdot{ }^{67,81-86,87}$ Crudely estimating the turbulent diffusivity $D_{\text {turb }} \sim \tilde{v}_{E}^{2} \tau_{\text {ac }}$ with autocorrelation time $\tau_{\text {ac }} \sim 1 / k_{\perp} \tilde{v}_{E}$ for $\tilde{v}_{E}$ the magnitude of the fluctuating portion of the $\mathbf{E} \times \mathbf{B}$ drift $\mathbf{v}_{E}$, one concludes that $D_{\text {turb }} \propto \tilde{\phi} / B$ should also decrease with increasing $r$, on the same scale length. To estimate the importance of collisional effects, multiply the pedestal-top thermal ion collision rate $\left.\nu_{i i}\right|_{\mathrm{pt}}$ by an ion crossing time $\tau_{c}$ defined as the pedestal ion stored energy over the ion heat flux, getting numbers around 1 for typical AUG, JET, and DIII-D parameters. Since collisionality scales as $v^{-3}$, superthermal pedestal-top ions typically escape to the SOL without experiencing a single collision, while subthermal pedestal-top ions have one or more. It will be shown that somewhat superthermal pedestal-top ions dominate the spin-up mechanism discussed here, so a collisionless approximation appears reasonable, at least for qualitative modeling. Given these typical edge orderings, the present work treats a model axisymmetric driftkinetic transport problem for ions. Collisions, parallel acceleration, $E_{r}$ shear, $\mathbf{E} \times \mathbf{B}$ divergence, magnetic trapping, and nondiffusive transport are all neglected. However, the model geometry incorporates both a pedestal and SOL region, which are treated on equal footing. The
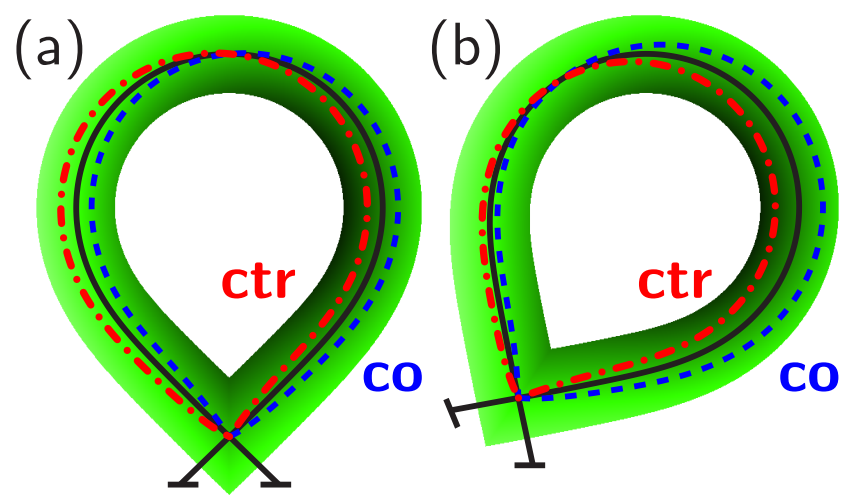

Figure 1. Drift orbits of co- and counter-current passing ions, sketched over shading that indicates the strength of the turbulent diffusivity, darker being stronger, for a straight-down (a) or major-radially inboard (b) X-point.

transport is modeled with a spatially dependent turbulent diffusivity, taken to be velocity-independent since $k_{\|} v_{t i} \ll \omega \sim c_{s} / L_{\perp}$, in which $c_{s} \sim v_{t i}$ since $T_{e} \sim T_{i}$. Although this approximation also neglects FLR effects, a finite passing-ion drift-orbit width is retained.

The resulting model leads to a remarkably simple physical picture of intrinsic rotation. ${ }^{88}$ Consider passing ions in the model pedestal, streaming freely along closed drift orbits with unchanging parallel velocity $v_{\|}$, while diffusing radially due to the fluctuating $\mathbf{E} \times \mathbf{B}$ drift. Regardless of the orientation of the toroidal field and plasma current, the drift orbits of co-current ions are shifted major-radially outwards, while those of countercurrent ions are shifted inwards. For the typical case of outboard-ballooning fluctuations, ${ }^{89}$ this implies that the orbit-averaged diffusivity experienced by co-current ions is smaller than that of counter-current ions, as sketched in Fig. 1(a). A nonrotating plasma therefore loses more counter-current ions than co-current ions, thus begins to rotate in the co-current direction. Since toroidal rotation damping is small, the rate of co-current bulk rotation must increase until its outward diffusion causes enough co-current momentum loss to balance out the countercurrent loss due to the $v_{\|}$-asymmetric orbit-averaged diffusivity, determining the pedestal-top intrinsic rotation velocity. Also, as sketched in Fig. 1(b), a major-radially inboard (outboard) X-point causes a net relative minorradial outward (inward) displacement of co-current ions, which leads in a fully analogous fashion to a co-current (counter-current) increment in the intrinsic rotation.

The rest of the paper is organized as follows. In Sec. II, the model equations will be presented and simplified with variable transformations, leading to a family of identical equations varying only in the value of a single parameter, the effective diffusivity $D_{\text {eff. }}$. Mathematical properties of the simplified equation and an approximate large- $D_{\text {eff }}$ solution are derived in Sec. III, while a small- $D_{\text {eff }}$ solution is obtained in Sec. IV. The corresponding pedestal-top rotation is derived and discussed in Sec. V. Extensions 
to and limitations of the model are discussed in Sec. VI. Sec. VII summarizes the paper.

\section{MODEL AND TRANSFORMATIONS}

In accord with the edge orderings already discussed, this paper analyzes and approximately solves a model axisymmetric drift-kinetic transport equation for the ions. The model, obtained in detail in App. A, represents a diffusive mean-field approximation to a steep-gradient, large-safety factor, large-aspect ratio, sub-sonic reduction of the standard collisionless, electrostatic gyrokinetic formulation of $\mathrm{Hahm},{ }^{90}$ set in a shearless, radially thin, simple-circular magnetic geometry $\mathbf{B}=\left(B_{\theta} b_{\theta} \hat{\theta}+\right.$ $\left.B_{\phi} b_{\phi} \hat{\phi}\right) R_{0} / R$ for constant magnitudes $B_{\phi}, B_{\theta}$, and $R_{0}$ and signs $b_{\phi}$ and $b_{\theta}$, letting $B_{0} \doteq\left(B_{\theta}^{2}+B_{\phi}^{2}\right)^{1 / 2}$. Neglecting the $\nabla B$ drift until Sec. VI and noting that $b_{\theta}$ is equal to the sign of the toroidal plasma current, Eq. (A3) may be written in normalized variables as

$\partial_{t} f_{i}+b_{\phi} v \partial_{y} f_{i}-b_{\phi} \delta v^{2}(\sin y) \partial_{x} f_{i}-D(y) \partial_{x}\left(e^{-x} \partial_{x} f_{i}\right)=0$.

Radial position $x$, poloidal position $y$, and time $t$ are respectively normalized to $L_{\phi}$, the minor radius $a$, and the pedestal-top ion thermal transit time $a B_{0} /\left.B_{\theta} v_{t i}\right|_{\mathrm{pt}}$, with $y=0$ at the outboard midplane. The physics retained is very basic: The axisymmetric ion parallel distribution function $f_{i}(x, y, v, t)$, normalized to pedestaltop ion density over thermal speed $\left.n_{i}\right|_{\mathrm{pt}} /\left.v_{t i}\right|_{\mathrm{pt}}$, is advected poloidally by the parallel velocity $v$, normalized to $\left.v_{t i}\right|_{\mathrm{pt}}$ and defined positive for co-current motion, and radially by the geodesic curvature drift. The importance of the curvature drift is indicated by the dimensionless parameter $\left.\delta \doteq q \rho_{i}\right|_{\mathrm{pt}} / L_{\phi}$, with $q \doteq a B_{\phi} / R_{0} B_{\theta}$ the safety factor, $\rho_{i} \doteq v_{t i} / \Omega_{i}$ the thermal ion gyroradius, and $\Omega_{i} \doteq e B_{0} / m_{i} c$. $\delta$ takes values around $1 / 4$ for typical ASDEX Upgrade (AUG) H-mode parameters. ${ }^{91}$ To model transport due to the nonaxisymmetric fluctuating $\mathbf{E} \times \mathbf{B}$ drift, a spatially inhomogeneous radial turbulent diffusivity is introduced, normalized to $\left.L_{\phi}^{2} B_{\theta} v_{t i}\right|_{\mathrm{pt}} / a B_{0}$. In the interests of analytical tractability, the diffusivity's spatial dependence is assumed separable, radially varying as $\exp (-x)$ with a strictly positive but otherwise arbitrary poloidal dependence $D(y)>0$. The domain is divided into a confined edge region $x \leq 0$ and SOL $x>0$. In the edge, the boundary conditions are poloidal periodicity $f_{i}\left(x \leq 0, y_{0}\right)=f_{i}\left(x \leq 0, y_{0}+2 \pi\right)$ and approach to a constant in the core $f_{i}(x \rightarrow-\infty, y) \rightarrow f_{i 0}(v) \geq 0$. In the SOL, the plasma vanishes at large minor radius $f_{i}(x \rightarrow \infty, y) \rightarrow 0$ and flows purely outward to the divertor legs, $f_{i}\left(x>0, y_{0}, b_{\phi} v>0\right)=0$ and $f_{i}(x>$ $\left.0, y_{0}+2 \pi, b_{\phi} v<0\right)=0$, with $y_{0}$ the poloidal angle of the X-point. Since $f_{i}$ is taken axisymmetric, Eq. (1) is invariant to a rigid toroidal rotation $v_{\text {rig }}$, normalized to $\left.v_{t i}\right|_{\mathrm{pt}} B_{\phi} / B_{0}$ and taken positive for co-current rotation. As may be trivially verified, Eq. (1) conserves particles $\int f_{i} d v$, a toroidal angular momentum $\int\left(v+v_{\text {rig }}\right) f_{i} d v$, and an energy $\int\left(1+v^{2} / 2\right) f_{i} d v$, in which the 1 represents perpendicular thermal energy. However, all of these quantities may flow into the domain from the left-hand side, physically representing particle, momentum and energy flux from the core, and out to the divertor cut, physically representing outflow to the divertor legs.

Due to the neglect of parallel acceleration, Eq. (1) may be solved velocity by velocity, treating $v$ as a parameter. This allows great simplification through a set of straightforward $v$-dependent variable transformations. First, switch the radial variable from a magnetic surface label to an ion drift-surface label,

$$
\bar{x} \doteq x-\delta v\left(\cos y-\cos y_{0}\right)
$$

obtaining an equation for $f_{i}(\bar{x}, y, v, t)$,

$$
\partial_{t} f_{i}+b_{\phi} v \partial_{y} f_{i}-D(y) e^{-\delta v\left(\cos y-\cos y_{0}\right)} \partial_{\bar{x}}\left(e^{-\bar{x}} \partial_{\bar{x}} f_{i}\right)=0 \text {. }
$$

Next, use a diffusivity-weighted poloidal coordinate

$$
\begin{aligned}
\bar{y} & \doteq D_{y 0}^{-1} \int_{y_{0}}^{y} D\left(y^{\prime}\right) e^{-\delta v\left(\cos y^{\prime}-\cos y_{0}\right)} d y^{\prime}, \\
D_{y 0}(v) & \doteq \int_{y_{0}}^{y_{0}+2 \pi} D\left(y^{\prime}\right) e^{-\delta v\left(\cos y^{\prime}-\cos y_{0}\right)} d y^{\prime},
\end{aligned}
$$

and restrict consideration to the steady-state problem $\partial_{t} f_{i}=0$, for which $f_{i}(\bar{x}, \bar{y}, v)$ must then satisfy

$$
b_{\phi} v \partial_{\bar{y}} f_{i}=D_{y 0} \partial_{\bar{x}}\left(e^{-\bar{x}} \partial_{\bar{x}} f_{i}\right) \text {. }
$$

Finally, apply the transformation $\bar{y} \rightarrow 1-\bar{y}$ for all $v$ satisfying $b_{\phi} v<0$ and switch to the distended radial variable

$$
u \doteq e^{\bar{x} / 2}
$$

obtaining an equation for $f_{i}(u, \bar{y}, v)$,

$$
\partial_{\bar{y}} f_{i}=\frac{1}{4} D_{\mathrm{eff}}\left(\partial_{u}^{2} f_{i}-\frac{1}{u} \partial_{u} f_{i}\right),
$$

in which $D_{\text {eff }} \doteq D_{y 0} /|v|$. Eq. (8) takes the transformed boundary conditions $f_{i}(u \leq 1,0, v)=f_{i}(u \leq 1,1, v)$, $f_{i}(0, \bar{y}, v)=f_{i 0}(v), f_{i}(u \rightarrow \infty, \bar{y}, v) \rightarrow 0, f_{i}(u>$ $1,0, v)=0$.

The principal goal of this calculation is to obtain the total radial flux of particles with each parallel velocity $v$. Eq. (1) may be written in standard continuity-equation form $\partial_{t} f_{i}(x, y, v)+\nabla \cdot \boldsymbol{\Gamma}=0$, with the dimensionless flux density

$$
\boldsymbol{\Gamma} \doteq b_{\phi}\left(\hat{y} v-\hat{x} \delta v^{2} \sin y\right) f_{i}-\hat{x} D(y) e^{-x} \partial_{x} f_{i},
$$

normalized to $\left.n_{i}\right|_{\mathrm{pt}} L_{\phi} B_{\theta} / a B_{0}$ in the $\hat{x}$ direction and $\left.n_{i}\right|_{\mathrm{pt}} B_{\theta} / B_{0}$ in the $\hat{y}$ direction. In steady state, Eq. (1) therefore implies that $\boldsymbol{\Gamma}$ is divergence-free, so the total outward flux of particles with velocity $v$ may be evaluated using any closed poloidal contour. The simplest form is obtained by evaluating the flux through an ion 
drift surface, defined parametrically in $x$ via the function $g_{x}(y) \doteq x_{0}+\delta v\left(\cos y-\cos y_{0}\right)$ for any constant $x_{0} \leq 0$, equivalently as a surface of constant $\bar{x} \leq 0$. The total dimensionless outward flux of particles with velocity $v$ is then simply

$\Gamma(v) \doteq \oint d \mathbf{A} \cdot \boldsymbol{\Gamma}=-\int_{y_{0}}^{y_{0}+2 \pi} D(y) e^{-g_{x}(y)} \partial_{x} f_{i}\left(g_{x}(y), y\right) d y$

normalized to $\left.n_{i}\right|_{\mathrm{pt}} L_{\phi} L_{\mathrm{tor}} B_{\theta} / B_{0}$, with $L_{\text {tor }} \doteq 2 \pi R_{0}$. Changing to the independent variable pair of Eq. (8) causes a simplification due to $\partial_{y} \bar{y}=$ $\operatorname{sign}\left(b_{\phi} v\right) D_{y 0}^{-1} D(y) \exp \left(-\delta v\left(\cos y-\cos y_{0}\right)\right)$, resulting in

$$
\Gamma(v)=-\frac{1}{2} D_{y 0} u^{-1} \int_{0}^{1} \partial_{u} f_{i} d \bar{y}
$$

evaluated at constant $u=\exp \left(x_{0} / 2\right) \leq 1$.

Eqs. (8) and (11) display a remarkable simplification. The original problem Eq. (1) has been exactly reduced to a one-parameter family of otherwise-identical differential equations, Eq. (8). The single parameter $D_{\text {eff }}$ represents an effective orbit-averaged turbulent diffusivity, which depends not only on the magnitude of $v$, but also on its sign! As sketched in Fig. 1 and discussed in the introduction, this follows from the fact that the major-radial orbit shifts of co- and counter-current passing ions are oppositely directed. For the typical case of outboard-ballooning and radially decreasing diffusivity, this implies that co-current ions effectively experience a weaker turbulent diffusion, since they are shifted minorradially outwards at the outboard midplane, thus avoiding the strongest turbulent diffusion. As will be evaluated in Secs. III-V, this results in a preferential exhaust of counter-current momentum, leaving the plasma to spin up in the co-current direction.

\section{EXACT AND LARGE- $D_{\text {eff }}$ ANALYSIS}

Eq. (8) resembles the "kinetic SOL" models of earlier works, ${ }^{92,93}$ which were solved using the Wiener-Hopf technique following Ref. 94. However, the procedure of Ref. 94 assumes a spatially homogeneous differential operator, thus cannot be applied to the explicitly $u$ dependent diffusion operator of Eq. (8). Fortunately, despite its inhomogeneity, the differential operator of Eq. (8) does possess an exact Green's function, which will be determined in this section, then used to prove existence and uniqueness of the solution for Eq. (8) and to derive an iterative scheme with strict error bounds for both $f_{i}$ and $\Gamma$, exhibiting rapid convergence for large $D_{\text {eff. }}$ Efficient treatment of the small- $D_{\text {eff }}$ case requires a different solution technique, which will be described in Sec. IV. Since solutions at different $v$ are independent in this model, the explicit $v$-dependence is suppressed in this section and in Sec. IV.
Taking an approach similar to Farnell and Gibson, ${ }^{95}$ one may obtain an exact Green's function for Eq. (8). The Green's function $G(u, \xi, \bar{y})$ should satisfy the homogeneous equation

$$
\partial_{\bar{y}} G-\frac{1}{4} D_{\mathrm{eff}}\left(\partial_{u}^{2} G-\frac{1}{u} \partial_{u} G\right)=0,
$$

subject to the boundary conditions $G(u, \xi, 0)=\delta(u-\xi)$, $G(0, \xi, \bar{y})=0$, and $G(u \rightarrow \infty, \xi, \bar{y}) \rightarrow 0$. Laplace transforming in $\bar{y}$ yields an equation for $G(u, \xi, s)=$ $\mathcal{L} G(u, \xi, \bar{y}) \stackrel{\doteq}{\doteq} \int_{0}^{\infty} \exp (-s \bar{y}) G(u, \xi, \bar{y}) d \bar{y}$

$$
s G-\frac{1}{4} D_{\mathrm{eff}}\left(\partial_{u}^{2} G-\frac{1}{u} \partial_{u} G\right)=\delta(u-\xi),
$$

subject to $G(0, \xi, s)=0$ and $G(u \rightarrow \infty, \xi, s) \rightarrow 0$. Defining $z \doteq 2\left(s / D_{\text {eff }}\right)^{1 / 2} u$, in which the root with positive real part is taken, the two homogeneous solutions of Eq. (13) may be written in terms of modified Bessel functions as $z I_{1}(z)$ and $z K_{1}(z)$, as is easily verified using Eqs. 9.6.27-28 of Abramowitz and Stegun (AS). ${ }^{96}$ To match the boundary conditions in $u$, the Green's function must then take the form $G(u, \xi, s)=H(\xi-$ u) $g_{L}(\xi, s) z I_{1}(z)+H(u-\xi) g_{R}(\xi, s) z K_{1}(z)$, with $H$ the step function. Choosing $g_{L}$ and $g_{R}$ so that $G$ is continuous at $u=\xi$ while $\left.\partial_{u} G\right|_{u=\xi_{+}}-\left.\partial_{u} G\right|_{u=\xi_{-}}=-4 / D_{\text {eff }}$, and noting AS Eq. 9.6.15, the Laplace-transformed Green's function is then uniquely determined to be

$G(u, \xi, s)=\frac{4 u}{D_{\mathrm{eff}}} K_{1}\left(\sqrt{\frac{4 s}{D_{\mathrm{eff}}}} \max (u, \xi)\right) I_{1}\left(\sqrt{\frac{4 s}{D_{\mathrm{eff}}}} \min (u, \xi)\right)$

The inverse Laplace transform, a special case of Eq. (A.8) of Ref. 95, Eq. (13.96) of Ref. 97, or Eq. (53) of Stix ${ }^{98}$ Ch. 10, ${ }^{99}$ yields the Green's function

$$
G(u, \xi, \bar{y})=\frac{2 u}{D_{\mathrm{eff}} \bar{y}} \exp \left(-\frac{u^{2}+\xi^{2}}{D_{\mathrm{eff}} \bar{y}}\right) I_{1}\left(\frac{2 u \xi}{D_{\mathrm{eff}} \bar{y}}\right) .
$$

The large-argument asymptotic approximation $I_{1}(w) \approx$ $e^{w} /(2 \pi w)^{1 / 2}$ (AS Eq. 9.7.1) may be used to verify that $G(u, \xi, \bar{y})$ indeed approaches $\delta(u-\xi)$ for $\bar{y} \rightarrow 0$, via $(u / \xi)^{1 / 2} \exp \left(-(u-\xi)^{2} / D_{\text {eff }} \bar{y}\right) /\left(\pi D_{\text {eff }} \bar{y}\right)^{1 / 2}$.

The Green's function given by Eq. (15) provides an integral form for the solution to Eq. (8), given any appropriately integrable specified values for $f_{i}(u, 0)$. However, since $G$ vanishes at $u=0$ while $f_{i}(0, \bar{y})=f_{i 0}$ is generally nonzero, this integral form must be applied to $f_{i}-f_{i 0}$ :

$$
f_{i}(u, \bar{y})-f_{i 0}=\int_{0}^{\infty} G(u, \xi, \bar{y})\left[f_{i}(\xi, 0)-f_{i 0}\right] d \xi
$$

Using Eq. 6.618.4 of Ref. 100, rewritten using AS Eq. 10.2.13 as

$$
\int_{0}^{\infty} G(u, \xi, \bar{y}) d \xi=1-e^{-u^{2} / D_{\mathrm{eff}} \bar{y}}
$$


and incorporating the boundary condition $f_{i}(u>1,0)=$ 0 allows the Green's function formula to be rewritten as

$$
f_{i}(u, \bar{y})=f_{i 0} e^{-u^{2} / D_{\mathrm{eff}} \bar{y}}+\int_{0}^{1} G(u, \xi, \bar{y}) f_{i}(\xi, 0) d \xi .
$$

Assuming bounded, continuous initial conditions $f_{i}(0 \leq$ $u \leq 1,0)$, the formula given by Eq. (18) is continuous ${ }^{101}$ and solves Eq. (8) for $\bar{y}>0$, with the additional boundary conditions $f_{i}(0, \bar{y})=f_{i 0}, f_{i}(u \rightarrow \infty, \bar{y}) \rightarrow 0, f_{i}(u>$ $1,0)=0$, as may be verified by direct substitution. ${ }^{102}$ Under reasonable requirements, this solution is shown to be unique in Appendix B.

To this point, the final boundary condition $f_{i}(u \leq 1,0)=f_{i}(u \leq 1,1)$ has not been addressed. To do this, one may use Eq. (18) to recast the problem in an integral form. Define an operator

$$
\mathcal{F}_{\bar{y}}[\psi] \doteq f_{i 0} e^{-u^{2} / D_{\mathrm{eff}} \bar{y}}+\int_{0}^{1} G(u, \xi, \bar{y}) \psi(\xi) d \xi,
$$

for all $\bar{y}>0$. Consider the special case $\mathcal{F}_{1}$, taken now to map a continuous 1 -D function on $[0,1]$ to a continuous 1 -D function on $[0,1]$. If one can find a $\bar{\psi}$ such that $\mathcal{F}_{1}[\bar{\psi}]=\bar{\psi}$, then $\mathcal{F}_{\bar{y}}[\bar{\psi}]$ solves Eq. (8) with all the original boundary conditions. Importantly, $\mathcal{F}_{1}$ (more generally, any $\mathcal{F}_{\bar{y}}$ ) represents a contraction mapping, meaning that for any continuous functions $\psi_{1}$ and $\psi_{2}$ on $[0,1]$, it is true that $\sup \left|\mathcal{F}_{1}\left[\psi_{2}\right]-\mathcal{F}_{1}\left[\psi_{1}\right]\right| \leq c \sup \left|\psi_{2}-\psi_{1}\right|$ for some Lipschitz constant $c<1$ : Noting that $G$ is nonnegative, one has

$$
\begin{array}{r}
\left|\mathcal{F}_{1}\left[\psi_{2}\right]-\mathcal{F}_{1}\left[\psi_{1}\right]\right|=\left|\int_{0}^{1} G(u, \xi, 1)\left[\psi_{2}(\xi)-\psi_{1}(\xi)\right] d \xi\right| \\
\leq\left[\int_{0}^{1} G(u, \xi, 1) d \xi\right] \sup \left|\psi_{2}(\xi)-\psi_{1}(\xi)\right| . \quad(20)
\end{array}
$$

Since $\int_{0}^{1} G(u, \xi, 1) d \xi \leq \int_{0}^{\infty} G(u, \xi, 1) d \xi$, Eq. (17) implies that $\mathcal{F}_{1}$ is a contraction mapping for all $D_{\text {eff }}>0$, with $c=1-\exp \left(-1 / D_{\text {eff }}\right)$. For $D_{\text {eff }} \geq 1$, recalling $u \leq 1$, one may use the facts that $I_{0}$ is nondecreasing and $\left|I_{0}\left(2 / D_{\text {eff }}\right)-1\right| \leq\left(I_{0}(2)-1\right) / D_{\text {eff }}^{2},{ }^{103}$ to show that

$$
\begin{aligned}
& \int_{0}^{1} G(u, \xi, 1) d \xi \leq \int_{0}^{1} \frac{2 u}{D_{\mathrm{eff}}} I_{1}\left(\frac{2 u \xi}{D_{\mathrm{eff}}}\right) d \xi \\
&=I_{0}\left(\frac{2 u}{D_{\mathrm{eff}}}\right)-1 \leq \frac{1}{D_{\mathrm{eff}}^{2}}\left[I_{0}(2)-1\right]
\end{aligned}
$$

in which $I_{0}(2)-1<1.28$, thus one has the additional Lipschitz constant $c=1.28 / D_{\text {eff }}^{2}$. Since $1-\exp \left(-1 / D_{\text {eff }}\right)$ is less than $1.28 / D_{\text {eff }}^{2}$ for $D_{\text {eff }}<1$, one may simply use

$$
c=\min \left[1-\exp \left(-1 / D_{\mathrm{eff}}\right), 1.28 / D_{\mathrm{eff}}^{2}\right]
$$

for all $D_{\text {eff. }}$.

Since $\mathcal{F}_{1}$ represents a contraction mapping for continuous functions on $[0,1]$, the Banach fixed point theorem implies that there is one and only one continuous function $\bar{\psi}$ on $[0,1]$ satisfying $\mathcal{F}_{1}[\bar{\psi}]=\bar{\psi}$. Combined with Appendix B, this implies existence and uniqueness of the solution to Eq. (8) with the original boundary conditions. ${ }^{104}$ The solution over all $\bar{y}>0$ is simply $\mathcal{F}_{\bar{y}}[\bar{\psi}]$, thus is continuous for all $\bar{y}>0$, while the boundary conditions then require continuity on $u \in[0,1), \bar{y}=0$ and $u>1, \bar{y}=0$. For the nontrivial problem $f_{i 0}>0$, the solution must however have a jump discontinuity at the single remaining point $u=1, \bar{y}=0$ (the $\mathrm{X}$-point), since Eq. (18) implies that $\bar{\psi}(1) \geq f_{i 0} \exp \left(-1 / D_{\text {eff }}\right)>0=$ $\lim _{u \rightarrow 1_{+}} f_{i}(u, 0)$. This fact will be important for correct calculation of the small- $D_{\text {eff }}$ limit in Sec. IV.

One may apply the above arguments to directly construct an iterative approximation and strictly bound its error. Start with any initial guess $\psi_{0}$ and let $\psi_{j \pm 1} \doteq$ $\mathcal{F}_{1}\left[\psi_{j}\right], \epsilon_{j} \doteq \psi_{j}-\bar{\psi}$, and $\epsilon_{j}^{\max } \doteq \sup \left|\epsilon_{j}\right|$, with $\bar{\psi}$ the unique solution of $\mathcal{F}_{1}[\bar{\psi}]=\bar{\psi}$. Application of Eq. (20) to $\left|\epsilon_{j+1}\right|=\left|\mathcal{F}_{1}\left[\psi_{j}\right]-\mathcal{F}_{1}[\bar{\psi}]\right|$ then implies that $\epsilon_{j+1}^{\max } \leq$ $c \epsilon_{j}^{\max } \leq c^{j+1} \epsilon_{0}^{\max }$, thus iterative mapping on any initial guess $\psi_{0}$ eventually approaches the fixed point $\bar{\psi}$. Eq. (17) then implies that the approximate solution resulting from $\psi_{j}, f_{i}^{(j)}(u, \bar{y}) \doteq \mathcal{F}_{\bar{y}}\left[\psi_{j}\right]$, also has absolute error strictly bounded by $\epsilon_{j}^{\max }$. Since $G$ is nonnegative, a nonnegative (resp. nonpositive) initial error $\epsilon_{0}$ leads to $\epsilon_{j}$ that are all nonnegative (resp. nonpositive).

The mere existence of this iterative solution procedure has two simple but important implications for the solution, $0 \leq f_{i} \leq f_{i 0}$ and $\partial_{u} f_{i} \leq 0$ : For $0 \leq f_{i} \leq f_{i 0}$, first note that the mapping $\mathcal{F}_{\bar{y}}[\psi]$, when applied to a $\psi$ satisfying $0 \leq \psi \leq f_{i 0}$, results by Eq. (17) in a $f_{i}$ satisfying $0 \leq f_{i}(u, \bar{y}) \leq f_{i 0}$. Next, since $\mathcal{F}_{1}$ is just the $\bar{y}=1$ case of $\mathcal{F}_{\bar{y}}$, one may immediately conclude that $0 \leq \psi_{j} \leq f_{i 0}$ implies $0 \leq \psi_{j+1} \leq f_{i 0}$. Since the series of functions $\psi_{j}$ generated by iterative mapping on any initial function $\psi_{0}$, including one satisfying $0 \leq \psi_{0} \leq f_{i 0}$, approaches the fixed point $\bar{\psi}$ uniformly, one may conclude that the true solution $f_{i}=\mathcal{F}_{\bar{y}}[\bar{\psi}]$ also satisfies $0 \leq f_{i} \leq f_{i 0}$. By identical logic, the fact that $\mathcal{F}_{\bar{y}}$ maps nonincreasing functions taking values between 0 and $f_{i 0}$ to nonincreasing functions (see App. C) implies that the true solution $f_{i}$ is nonincreasing.

The $u$ partial of the exact and approximate solutions are given simply by

$$
\partial_{u} \mathcal{F}_{\bar{y}}[\psi]=-\frac{2 u}{D_{\mathrm{eff}} \bar{y}} f_{i 0} e^{-u^{2} / D_{\mathrm{eff}} \bar{y}}+\int_{0}^{1} \psi \partial_{u} G d \xi,
$$

with $\psi$ either the fixed point $\bar{\psi}$ or any approximation $\psi_{j}$, thus the error in the $u$ partial may be bounded by

$$
\left|\partial_{u} f_{i}^{(j)}-\partial_{u} f_{i}\right|=\left|\int_{0}^{1}\left(\psi_{j}-\bar{\psi}\right) \partial_{u} G d \xi\right| \leq \epsilon_{j}^{\max } \int_{0}^{1}\left|\partial_{u} G\right| d \xi .
$$

The Green's function's $u$ partial,

$$
\partial_{u} G=\frac{4 u}{D_{\mathrm{eff}}^{2} \bar{y}^{2}} e^{-\left(u^{2}+\xi^{2}\right) / D_{\mathrm{eff}} \bar{y}}\left[\xi I_{0}\left(\frac{2 u \xi}{D_{\mathrm{eff}} \bar{y}}\right)-u I_{1}\left(\frac{2 u \xi}{D_{\mathrm{eff}} \bar{y}}\right)\right],
$$




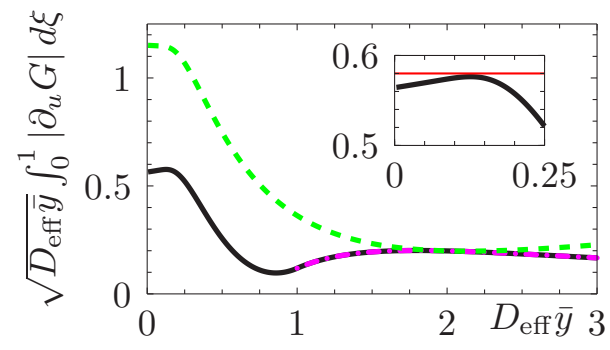

Figure 2. Bounds for $\sqrt{D_{\text {eff }} \bar{y}} \int_{0}^{1}\left|\partial_{u} G\right| d \xi$ evaluated at $u=1$, used to bound the error in $\Gamma$. The analytical formulas of Eqs. (27) (dash-dotted purple) and (28) (dashed green) may be compared with the numerical evaluation leading to Eq. (29) (solid black), while the inset also shows the inferred upper bound 0.58 (thin red).

is exactly integrable in $\xi$ since $\partial_{\xi} G_{I}=-\partial_{u} G$ for

$$
G_{I}(u, \xi, \bar{y}) \doteq \frac{2 u}{D_{\text {eff }} \bar{y}} e^{-\left(u^{2}+\xi^{2}\right) / D_{\text {eff }} \bar{y}} I_{0}\left(\frac{2 u \xi}{D_{\text {eff }} \bar{y}}\right) .
$$

When $u^{2} \leq D_{\text {eff }} \bar{y}$, the inequality $w I_{0}(w) \geq 2 I_{1}(w)$ implies $\partial_{u} G \geq 0$ for all $\xi$, so we may use the exact integral, $I_{0}(w) \geq 1$, and $1-e^{-w} \leq w$ to obtain

$$
\begin{aligned}
\frac{u^{2}}{D_{\mathrm{eff}} \bar{y}} \leq 1: \int_{0}^{1}\left|\partial_{u} G\right| d \xi & =\frac{2 u e^{-u^{2} / D_{\mathrm{eff}} \bar{y}}}{D_{\mathrm{eff}} \bar{y}}\left[1-\frac{I_{0}\left(\frac{2 u}{D_{\text {eff }}}\right)}{e^{1 / D_{\mathrm{eff}} \bar{y}}}\right] \\
& \leq \frac{2 u e^{-u^{2} / D_{\mathrm{eff}} \bar{y}}}{D_{\mathrm{eff}} \bar{y} \max \left(1, D_{\mathrm{eff}} \bar{y}\right)} .
\end{aligned}
$$

To bound generally for all $u$ and $D_{\text {eff }} \bar{y}$, note that Appendix $\mathrm{C}$ shows that there is a $\xi_{0} \geq 0$ such that $\partial_{u} G<0$ for $0<\xi<\xi_{0}$ and $\partial_{u} G>0$ for $\xi>\xi_{0}$, which implies that $\int_{0}^{1}\left|\partial_{u} G\right| d \xi \leq 2 G_{I}\left(\xi_{0}\right)-\left[G_{I}(0)+G_{I}(1)\right]$. The function $\left(D_{\text {eff }} \bar{y}\right)^{1 / 2} G_{I}$, dependent only on $u /\left(D_{\text {eff }} \bar{y}\right)^{1 / 2}$ and $\xi /\left(D_{\text {eff }} \bar{y}\right)^{1 / 2}$, is bounded above by $\sqrt{2} e^{-1 / 2},{ }^{105}$ allowing us to conclude that

$$
\int_{0}^{1}\left|\partial_{u} G\right| d \xi \leq \frac{2 \sqrt{2} e^{-1 / 2}}{\left(D_{\mathrm{eff}} \bar{y}\right)^{1 / 2}}-\left[G_{I}(u, 0, \bar{y})+G_{I}(u, 1, \bar{y})\right] .
$$

Numerical evaluation, compared with Eqs. (27) and (28) in Fig. 2, shows that for the special case $u=1$ one gets

$$
u=1: \int_{0}^{1}\left|\partial_{u} G\right| d \xi \leq 0.58 / \sqrt{D_{\text {eff }} \bar{y}} .
$$

Since the true solution $f_{i}$ satisfies $0 \leq f_{i} \leq f_{i 0}$, the simple initial guess $\psi_{0}=0$ satisfies $\epsilon_{0}^{\max } \leq f_{i 0}$. The corresponding first-order solution

$$
\begin{aligned}
& f_{i}^{A}(u, \bar{y}) \doteq \mathcal{F}_{\bar{y}}\left[\mathcal{F}_{1}[0]\right] \\
& \quad=f_{i 0} e^{-u^{2} / D_{\mathrm{eff}} \bar{y}}+f_{i 0} \int_{0}^{1} G(u, \xi, \bar{y}) e^{-\xi^{2} / D_{\mathrm{eff}}} d \xi
\end{aligned}
$$

has absolute error strictly bounded by $c f_{i 0}$. One may therefore use Eqs. (22), (24), (27), and (29) to bound the absolute error in the corresponding flux estimate ${ }^{106,107}$

$$
\begin{aligned}
\Gamma^{A} & \doteq-\frac{1}{2} D_{y 0} u^{-1} \int_{0}^{1} \partial_{u} f_{i}^{A} d \bar{y} \\
& =f_{i 0}|v|\left[\ln \left(D_{\text {eff }}\right)-\gamma+2 / D_{\text {eff }}+O\left(D_{\text {eff }}^{-2}\right)\right]
\end{aligned}
$$

evaluated at $u=1$ and with $\gamma \approx 0.5772$ the Euler constant, with

$$
\begin{aligned}
& \left|\Gamma^{A}-\Gamma\right| / f_{i 0}|v| \leq \frac{1}{2}\left(D_{\text {eff }} / f_{i 0}\right) \int_{0}^{1}\left|\partial_{u} f_{i}^{A}-\partial_{u} f_{i}\right| d \bar{y} \\
\leq & \min \left[0.58 \sqrt{D_{\text {eff }}}\left(1-e^{-1 / D_{\text {eff }}}\right), 0.75 / D_{\text {eff }}^{3 / 2}, 1.21 / D_{\text {eff }}^{2}\right] .
\end{aligned}
$$

The first two bounds only use Eq. (29), while the third uses Eq. (27) for $\bar{y}>D_{\text {eff }}^{-1}$.

\section{SMALL- $D_{\text {eff }}$ ANALYSIS}

Although the iterative method of Sec. III is convergent for all $D_{\text {eff }}$, the rate of convergence becomes slow for $D_{\text {eff }} \ll 1$. In this limit, Eq. (8) may be solved separately in the edge and SOL, using a Fourier series for $u<1$ and a Laplace transform for $u>1$. Following Oldham and Spanier, ${ }^{108}$ the Laplace-transformed relation between $f_{i}$ and $\partial_{u} f_{i}$ may be approximately inverted for small $D_{\text {eff. }}$. The real-space relation may then be used to enforce continuity of $f_{i}$ and $\partial_{u} f_{i}$ at $u=1$ for all $\bar{y}>0$. The resulting dense matrix equation for the Fourier coefficients must be truncated and solved numerically, yielding the desired small- $D_{\text {eff }}$ relation between $D_{\text {eff }}$ and $\Gamma$. With this relation in hand, approximations to $\Gamma$ that are good for all $D_{\text {eff }}$ are finally identified.

To solve Eq. (8) for $u<1$, take $f_{i}(u, \bar{y})=$ $\sum_{m=-\infty}^{\infty} f_{m}(u) \exp (2 \pi i m \bar{y})$, obtaining ODEs for the $m \geq 0$ coefficients,

$$
f_{m}^{\prime \prime}(u)-\frac{1}{u} f_{m}^{\prime}(u)-\frac{8 \pi i m}{D_{\mathrm{eff}}} f_{m}(u)=0,
$$

with the reality constraints determining $f_{-m}=f_{m}^{*}$. Incorporating the boundary condition $f_{i}(0, \bar{y})=f_{i 0}$ and defining $z_{e, m} \doteq\left(8 \pi i m / D_{\text {eff }}\right)^{1 / 2} u$, Eq. (33) has the solutions $f_{0}(u)=f_{i 0}-c_{q} u^{2}$ and $f_{m}=c_{m} z_{e, m} I_{1}\left(z_{e, m}\right)$ for asyet-undetermined complex coefficients $c_{q}$ and $c_{m}$. This may be straightforwardly verified using AS Eqs. 9.6.2728 , which also show $f_{m}^{\prime}=c_{m} z_{e, m}^{2} I_{0}\left(z_{e, m}\right) / u$.

For $u>1$, Laplace-transform Eq. (8) in $\bar{y},{ }^{109}$ obtaining an equation for $F(u, s) \doteq \mathcal{L} f_{i}(u, \bar{y})$ :

$$
\partial_{u}^{2} F-\frac{1}{u} \partial_{u} F-\frac{4 s}{D_{\text {eff }}} F=0 .
$$

Incorporating the boundary condition $f_{i}(u \rightarrow \infty, \bar{y}) \rightarrow 0$ and defining $z_{S} \doteq 2 u \sqrt{s / D_{\text {eff }}}$ with positive real part, 
Eq. (34) has the solution $F=c_{S}(s) z_{S} K_{1}\left(z_{S}\right)$, with $c_{S}$ an as-yet-arbitrary function of $s$. AS Eqs. 9.6.27-28 may again be used to verify the solution, also yielding $\partial_{u} F=$ $-c_{S}(s) z_{S}^{2} K_{0}\left(z_{S}\right) / u$.

Since small $D_{\text {eff }}$ corresponds to large $z_{S}$, use AS Eq. 9.7.2 to obtain the asymptotic approximation to the ratio of $F$ and $\partial_{u} F$ :

$$
\frac{F}{\partial_{u} F}=-\frac{u}{z_{S}} \frac{K_{1}\left(z_{S}\right)}{K_{0}\left(z_{S}\right)} \stackrel{\left|z_{S}\right| \rightarrow \infty}{\sim}-\frac{u}{z_{S}}-\frac{u}{2 z_{S}^{2}}+\cdots
$$

Multiplying the asymptotic relation by $\partial_{u} F$, one may invert the Laplace transform using the convolution theorem $\left[\mathcal{L}\left(\int_{0}^{t} f(\tau) g(t-\tau) d \tau\right)=(\mathcal{L} f)(\mathcal{L} g)\right.$ for arbitrary functions $f(t)$ and $g(t)$ and the Laplace transform pairs $\mathcal{L}(\pi \bar{y})^{-1 / 2}=s^{-1 / 2}$ and $\mathcal{L}(1)=s^{-1}$, obtaining the $u>1$ relation

$$
f_{i} \approx-\frac{1}{2 \sqrt{\pi}} D_{\mathrm{eff}}^{1 / 2} \int_{0}^{\bar{y}} \frac{\partial_{u} f_{i}}{\sqrt{\bar{y}-y^{\prime}}} d y^{\prime}-\frac{1}{8 u} D_{\mathrm{eff}} \int_{0}^{\bar{y}} \partial_{u} f_{i} d y^{\prime} .
$$

As demonstrated in App. D, the true relation between $f_{i}$ and $\partial_{u} f_{i}$ lays between that given in Eq. (36) and the leading-order truncation [Eq. (36) omitting the last term], with the relative error of either approximation therefore vanishing as $D_{\text {eff }} \rightarrow 0$. Note that the formulation of Eq. (36) may be used even if $\lim _{\bar{y} \rightarrow 0} f_{i}(u, \bar{y}) \neq 0$, as in fact occurs for $u=1$ due to the jump discontinuity in $f_{i}$ at $u=1, \bar{y}=0$ discussed in Sec. III. Eq. (36) then implies that $\partial_{u} f_{i}$ has a corresponding $\bar{y}$-integrable singularity, with $\partial_{u} f_{i}(u=1, \bar{y} \rightarrow 0)$ asymptoting to $-2 /\left(\pi D_{\text {eff }} \bar{y}\right)^{1 / 2}$ times $\lim _{\bar{y} \rightarrow 0} f_{i}(1, \bar{y})$.

By continuity of $f_{i}$ and $\partial_{u} f_{i}$ at $u=1, \bar{y}>0$, the $u<1$ solution must satisfy Eq. (36) at $u=1$. Straightforward substitution of the Fourier series expansion into Eq. (36) at $u=1$ yields

$$
\begin{gathered}
f_{i 0}-c_{q}+\sum_{m \neq 0} f_{m} e^{2 \pi i m \bar{y}}-\frac{2}{\sqrt{\pi}} D_{\mathrm{eff}}^{1 / 2} c_{q} \sqrt{\bar{y}}-\frac{1}{4} D_{\mathrm{eff}} c_{q} \bar{y} \\
+\frac{1}{2} D_{\mathrm{eff}}^{1 / 2} \sum_{m \neq 0} f_{m}^{\prime} e^{2 \pi i m \bar{y}} \frac{\operatorname{erf}(\sqrt{2 \pi i m \bar{y}})}{\sqrt{2 \pi i m}} \\
+\frac{1}{8} D_{\mathrm{eff}} \sum_{m \neq 0} f_{m}^{\prime} \frac{e^{2 \pi i m \bar{y}}-1}{2 \pi i m} \approx 0
\end{gathered}
$$

with erf the error function. [The ratio $\operatorname{erf}\left((2 \pi i m \bar{y})^{1 / 2}\right) /(2 \pi i m)^{1 / 2}$ is independent of branch choice as long as the branch is chosen consistently for the two roots, a property that will hold for all similar square-root pairs in this section.]

Recall the goal: to calculate the $D_{\text {eff-dependent rela- }}$ tion between $f_{i 0}$ and $\Gamma$, the latter of which may be easily evaluated as $\Gamma=D_{y 0} c_{q}$. In principle, Eq. (37) must furnish $c_{q}$ as a function of $f_{i 0}$. However, since $f_{i 0}$ appears only in the $m=0$ component of Eq. (37), it is easier to take $c_{q}$ as given and solve for $f_{i 0}$, then invert the resulting scalar function $f_{i 0}\left(c_{q}\right)$ after the fact. Specifically, let the real and imaginary portions of the $m>0$ components of Eq. (37) define an inhomogeneous real matrix equation for the real and imaginary parts of the $m>0$ coefficients, $f_{m, r}^{\prime} \doteq \operatorname{Re} f_{m}^{\prime}$ and $f_{m, i}^{\prime} \doteq \operatorname{Im} f_{m}^{\prime}$, using the relation $f_{m}=\left[u I_{1}\left(z_{e, m}\right) / z_{e, m} I_{0}\left(z_{e, m}\right)\right] f_{m}^{\prime}$, obtaining

$$
\mathbf{M} \cdot\left(f_{1, r}^{\prime}, f_{2, r}^{\prime}, \cdots, f_{1, i}^{\prime}, f_{2, i}^{\prime}, \cdots\right) \approx c_{q} \mathbf{v}
$$

at which point the $m=0$ component may be written as

$$
\begin{aligned}
& f_{i 0}-\left(1+\frac{4}{3 \sqrt{\pi}} D_{\mathrm{eff}}^{1 / 2}+\frac{1}{8} D_{\mathrm{eff}}\right) c_{q} \\
\approx & D_{\mathrm{eff}}^{1 / 2} \mathbf{u} \cdot\left(f_{1, r}^{\prime}, f_{2, r}^{\prime}, \cdots, f_{1, i}^{\prime}, f_{2, i}^{\prime}, \cdots\right)=c_{q} D_{\mathrm{eff}}^{1 / 2} \Psi\left(D_{\mathrm{eff}}\right),
\end{aligned}
$$

in which the scalar function $\Psi\left(D_{\text {eff }}\right) \doteq \mathbf{u} \cdot \mathbf{M}^{-1} \cdot \mathbf{v}$ approaches a finite nonzero value for $D_{\text {eff }} \rightarrow 0$. Noting the relations $\operatorname{erf}\left((2 \pi i)^{1 / 2} w\right) /(2 i)^{1 / 2}=C(2 w)-i S(2 w)$ and $\operatorname{erf}\left((-2 \pi i)^{1 / 2} w\right) /(-2 i)^{1 / 2}=C(2 w)+i S(2 w)$, where the Fresnel integrals $C(w) \doteq \int_{0}^{w} \cos \left(\frac{1}{2} \pi t^{2}\right) d t$ and $S(w) \doteq$ $\int_{0}^{w} \sin \left(\frac{1}{2} \pi t^{2}\right) d t$ are odd and are purely real for real argument, one may obtain the vector and matrix components: $v_{m, r}=-S(2 \sqrt{m}) /(\pi m)^{3 / 2}, v_{m, i}=\left[2 / \sqrt{\pi}+D_{\mathrm{eff}}^{1 / 2} / 4-\right.$ $C(2 \sqrt{m}) / \sqrt{\pi m}] / \pi m, \quad u_{m, r}=S(2 \sqrt{m}) / 2(\pi m)^{3 / 2}$, $u_{m, i}=\left[2 / \sqrt{\pi}+D_{\mathrm{eff}}^{1 / 2} / 4-C(2 \sqrt{m}) / \sqrt{\pi m}\right] / 2 \pi m$, $M_{m r, m r}=c_{\mathrm{Re}}-S(2 \sqrt{m}) / 2(\pi m)^{3 / 2}, M_{m r, m i}=-c_{\mathrm{Im}}$, $M_{m i, m r}=c_{\mathrm{Im}}, M_{m i, m i}=c_{\mathrm{Re}}+S(2 \sqrt{m}) / 2(\pi m)^{3 / 2}$, with $c_{\operatorname{Re}} \doteq \operatorname{Re}\left(I_{1}\left(z_{e, m}\right) / \sqrt{2 \pi i m} I_{0}\left(z_{e, m}\right)\right)+$ $[C(2 \sqrt{m})+S(2 \sqrt{m}) / 4 \pi m] / \sqrt{\pi m}$ and $c_{\operatorname{Im}} \doteq$ $\operatorname{Im}\left(I_{1}\left(z_{e, m}\right) / \sqrt{2 \pi i m} I_{0}\left(z_{e, m}\right)\right)-\left(1 / \sqrt{\pi}+\frac{1}{4} D_{\text {eff }}^{1 / 2}\right) / 2 \pi m+$ $[C(2 \sqrt{m}) / 4 \pi m-S(2 \sqrt{m})] / \sqrt{\pi m}$, and for $n \neq m$ : $M_{m r, n r}=[\sqrt{m} S(2 \sqrt{m})-\sqrt{n} S(2 \sqrt{n})] / \pi^{3 / 2}\left(n^{2}-m^{2}\right)$, $M_{m r, n i}=[\sqrt{n} C(2 \sqrt{n})-n C(2 \sqrt{m}) / \sqrt{m}] /$ $\pi^{3 / 2}\left(n^{2}-m^{2}\right), \quad M_{m i, n r}=[-m C(2 \sqrt{n}) / \sqrt{n}+$ $\sqrt{m} C(2 \sqrt{m})] / \pi^{3 / 2}\left(n^{2}-m^{2}\right)$, and $M_{m i, n i}=$ $[n S(2 \sqrt{m}) / \sqrt{m}-m S(2 \sqrt{n}) / \sqrt{n}] / \pi^{3 / 2}\left(n^{2}-m^{2}\right)$.

Eqs. (38)-(39) have been truncated and numerically solved, obtaining $\Psi\left(D_{\text {eff }}\right)$ for values of $D_{\text {eff }}$ between 0 and $10^{4}$, retaining up to $m=10000$ in each case. The truncation-induced error in $\Psi$ was estimated with the quantity $\varepsilon$ obtained by evaluating the LHS of Eq. (37) for the approximate result, dividing by $c_{q} D_{\text {eff }}^{1 / 2}$, and averaging (rms) over $0 \leq \bar{y} \leq 1$. Fig. 3 shows $\Psi$ along with its error estimate and the least-squares fit $\Psi \approx$ $0.0701+0.0513 D_{\text {eff }}^{1 / 2}$, calculated using only values of $\Psi$ for $D_{\text {eff }} \leq 1$ since the asymptotic approximation becomes inaccurate for larger $D_{\text {eff. }}$ Using the fit in Eq. (39), one finally obtains the desired approximate small- $D_{\text {eff }}$ relation

$$
\Gamma \approx \Gamma^{a} \doteq f_{i 0}|v| \frac{D_{\mathrm{eff}}}{1+a_{1} D_{\mathrm{eff}}^{1 / 2}+a_{2} D_{\mathrm{eff}}}
$$

with $a_{1}=0.8224$ and $a_{2}=0.1763$.

The large- and small- $D_{\text {eff }}$ results for $\Gamma$ overlap quite well for for a range of intermediate $D_{\text {eff }}$ including some tight error bounds on the large- $D_{\text {eff }}$ solution [Eq. (32)], 


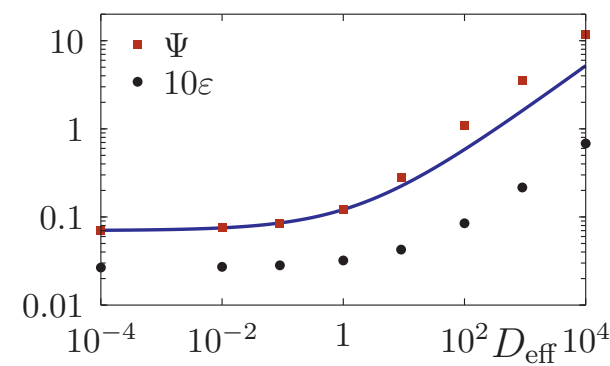

Figure 3. $\Psi\left(D_{\text {eff }}\right)$ versus $D_{\text {eff }}$, along with the fit (solid blue line) and 10 times the error estimate $\varepsilon$.

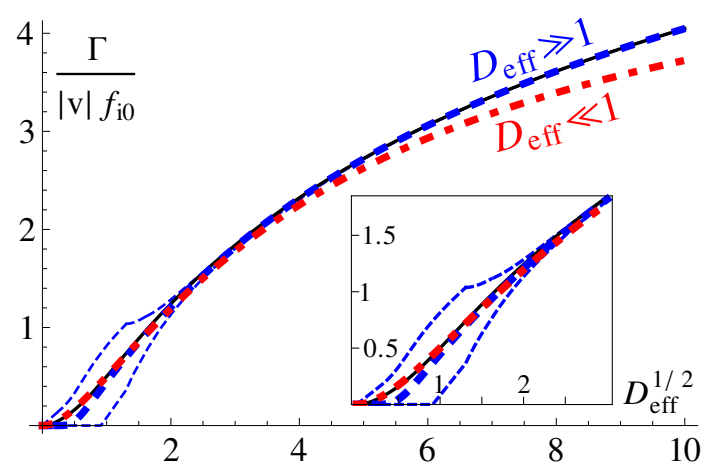

Figure 4. Large- $D_{\text {eff }}\left(\Gamma^{A} / f_{i 0}|v|\right.$, thick dashed blue) and small$D_{\text {eff }}\left(\Gamma^{a} / f_{i 0}|v|\right.$, thick dash-dotted red) approximations for $\Gamma / f_{i 0}|v|$, along with the large- $D_{\text {eff }}$ error bounds (thin dashed blue) and uniform approximation $\Gamma^{\ell} / f_{i 0}|v|$ (thin solid black).

as shown in Fig. 4, so one may splice them together to obtain an expression for $\Gamma$ good over all $D_{\text {eff }}$. Comparing Eqs. (31) and (40), one sees that any fitted approximation for $\Gamma / f_{i 0}|v|$ must asymptote to $\ln D_{\text {eff }}$ for large $D_{\text {eff }}$ and drop off linearly with $D_{\text {eff }} \rightarrow 0$. The simple form

$$
\Gamma^{s} \doteq f_{i 0}|v| \ln \left(1+e^{-\gamma} D_{\text {eff }}\right)
$$

does this, additionally capturing the constant term and approximating the $D_{\text {eff }}^{-1}$ term at large $D_{\text {eff }}$. One may improve the fit using the log of the higher-order polynomial

$$
\Gamma^{\ell} \doteq \frac{1}{4} f_{i 0}|v| \ln \left(1+\sum_{j=2}^{8} c_{j} D_{\mathrm{eff}}^{j / 2}\right),
$$

with $c_{2}=4$ and $c_{3}=-4 a_{1}$ chosen to match $\Gamma^{a}$ to order $D_{\text {eff }}^{3 / 2}$ for small $D_{\text {eff }} ; c_{5}=c_{7}=0, c_{6}=8 e^{-4 \gamma}$, and $c_{8}=$ $e^{-4 \gamma}$ to match $\Gamma^{A}$ to order $D_{\text {eff }}^{-3 / 2}$ for large $D_{\text {eff }}$; and $c_{4}=\exp \left(4 /\left(1+a_{1}+a_{2}\right)\right)+4 a_{1}-5-9 e^{-4 \gamma}$ to set $\Gamma^{\ell}$ equal to $\Gamma^{a}$ at $D_{\text {eff }}=1$.

\section{ROTATION}

In Secs. III and IV, explicit expressions for the velocitydependent flux $\Gamma(v)$ were determined in both large- and small- $D_{\text {eff }}$ approximations, then fitted with simple $\left(\Gamma^{s}\right)$ and more precise $\left(\Gamma^{\ell}\right)$ approximations to $\Gamma$ good for all $D_{\text {eff. }}$ In this section, these flux expressions will be combined with the assumption of a rigidly-rotating Maxwellian at the boundary with the core, $f_{i 0}(v)=$ $e^{-v^{2} / 2} / \sqrt{2 \pi}$ in the rotating frame, to obtain a general momentum balance equation determining the pedestaltop rotation velocity in the presence of an arbitrary specified torque on the core plasma. The special cases of intrinsic rotation and zero rotation will be evaluated and discussed. Although the general formulas may be used for any $D(y)>0$, plots and linearizations will assume a simple ballooning diffusivity $D(y)=D_{0}(1+$ $\left.d_{c} \cos y\right)$ with constant $D_{0}$ and $\left|d_{c}\right|<1$, for which AS Eq. 9.6.19 yields $D_{\text {eff }}(v)=2 \pi D_{0} \exp \left(\delta v \cos y_{0}\right)\left[I_{0}(\delta v)-\right.$ $\left.d_{c} I_{1}(\delta v)\right] /|v|$. Plots use the parameters $D_{0}=0.033$, $d_{c}=0.8, y_{0}=-5 \pi / 8$, and $\delta=0.28$, representative of AUG H-mode values. ${ }^{110}$ Numerical evaluation uses $\Gamma^{\ell}$ and analytical formulas use $\Gamma^{s}$.

The predicted total fluxes of density, toroidal angular momentum, and parallel heat may now be straightforwardly obtained as appropriate moments of $\Gamma$. In the rotating frame, these are

$$
\begin{aligned}
\Gamma^{p} & \doteq \int_{-\infty}^{\infty} \Gamma d v \approx \sqrt{\frac{2}{\pi}} g_{1} \\
\Pi & \doteq \int_{-\infty}^{\infty} v \Gamma d v \approx 8 \delta \sqrt{\frac{2}{\pi}}\left(\cos y_{0}-\frac{d_{c}}{2}\right)\left(g_{3}-g_{5}\right) \\
Q_{\|} & \doteq \int_{-\infty}^{\infty} \frac{v^{2}}{2} \Gamma d v \approx \sqrt{\frac{2}{\pi}} g_{3}
\end{aligned}
$$

respectively normalized to $\left.\left.n_{i}\right|_{\mathrm{pt}} v_{t i}\right|_{\mathrm{pt}} L_{\phi} L_{\mathrm{tor}} B_{\theta} / B_{0}$ times $1,\left.\quad m_{i} v_{t i}\right|_{\mathrm{pt}} R_{0} B_{\phi} / B_{0}$, and $\left.m_{i} v_{t i}\right|_{\mathrm{pt}} ^{2}$, and in which $g_{p}\left(D_{0}\right) \doteq \ln \left(1+2 \pi D_{0} / e^{\gamma} p^{1 / 2}\right)$. (Details of the linearization are given in App. E.) The integrands for $\Gamma^{p}, \Pi$, and $Q_{\|}$, plotted in Fig. 5, show the momentum and heat transport to be dominated by somewhat suprathermal pedestal-top ions. The momentum transport $\Pi$ is negative, indicating an outward flux of counter-current momentum, thus a co-current acceleration for a nonrotating plasma. Since $\Pi$ is independent of the rigid toroidal rotation or its gradient, it represents a residual stress. The fact that $\Pi$ is a significant fraction of $\Gamma^{p}$ and $Q_{\|}$ demonstrates the robustness of the mechanism and implies significant momentum transport for a nonrotating plasma.

In the lab frame, one obtains the general steady-state momentum balance equation

$$
\tau=\int_{-\infty}^{\infty}\left(v_{\text {rig }}+v\right) \Gamma d v=v_{\text {rig }} \Gamma^{p}+\Pi
$$

balancing the co-current torque $\tau$ applied to the plasma core, normalized to $\left.\left.n_{i}\right|_{\mathrm{pt}} T_{i}\right|_{\mathrm{pt}} L_{\phi} L_{\mathrm{tor}} R_{0} B_{\theta} B_{\phi} / B_{0}^{2}$, with the outward flux of co-current momentum through the pedestal. The central result of this article, Eq. (44) may be used to estimate pedestal-top rotation in the presence of some specified torque on the core (for which 


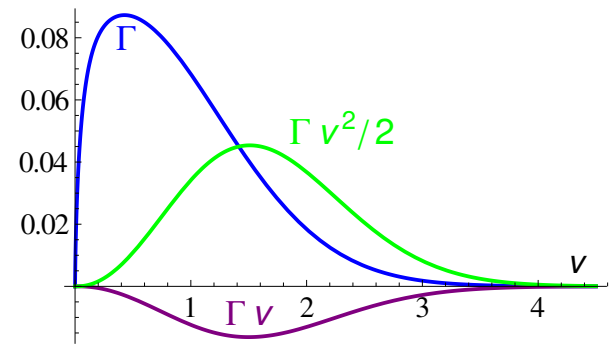

Figure 5. Integrands for $\Gamma^{p}, \Pi$, and $Q_{\|}$, summed over positive and negative $v$.

intrinsic rotation is a special case) or to infer the approximate torque required to obtain a specific pedestaltop rotation (for which null rotation is a special case). Being determined by a balance of fluxes through the pedestal, the pedestal-top rotation should settle to its steady-state value on the rather rapid pedestal transport timescale, a few times the ion transit time at the LCFS, $\sim q_{95} R_{0} /\left.v_{t i}\right|_{\text {sep }}$. Since the core rotation evolution is typically much slower, Eq. (44) may often be used to estimate the quasistatic response of pedestal-top rotation to a slowly changing momentum flux from the core. As an example, such an estimate could be used to provide an outer boundary condition for a global simulation of core momentum transport. When the predicted momentum flux from the core $(\tau)$ is small relative to $\Pi$, the edge rotation becomes effectively "stiff," approximately taking a fixed value regardless of $\tau$.

Intrinsic rotation is an important special case, defined by vanishing core torque $\tau$. In the absence of toroidal momentum sources and damping, the net outward flux of toroidal angular momentum through the pedestal must vanish. Since Eq. (1) is independent of $v_{\text {rig }}$, so are $\Gamma^{p}$ and $\Pi$, thus one may solve Eq. (44) trivially for the intrinsic rigid rotation $v_{\text {int }}$ :

$$
v_{\mathrm{int}}=-\frac{\Pi}{\Gamma^{p}} \approx 8 \delta\left(d_{c} / 2-\cos y_{0}\right) \frac{g_{3}-g_{5}}{g_{1}} .
$$

As shown in Fig. 6(a), the intrinsic rotation is co-current and represents a Mach number of up to a few tenths for realistic parameter values. The dimensional linearization in the small- $D_{0}$ limit,

$$
\left.v_{\text {int }}^{\operatorname{dim}} \approx 1.04 \frac{B_{\phi}}{B_{0}}\left(d_{c} / 2-\cos y_{0}\right) v_{t i}\right|_{\mathrm{pt}} \frac{\left.q \rho_{i}\right|_{\mathrm{pt}}}{L_{\phi}} \propto \frac{\left.T_{i}\right|_{\mathrm{pt}}}{L_{\phi} B_{\theta}},
$$

shows the basic physical scaling of the pedestal-top rotation velocity: linear in the product of the pedestaltop passing-ion drift-orbit width $\left.q \rho_{i}\right|_{\mathrm{pt}}$ and ion thermal speed $\left.v_{t i}\right|_{\mathrm{pt}}$. The drift orbit width introduces a $1 / B_{\theta} \propto$ $1 / I_{p}$ scaling, as typically observed in experiment, ${ }^{6,24}$ while the predicted linear dependence of edge rotation on temperature has been recently observed in dedicated experiments. ${ }^{10,15} \mathrm{~A}$ co-current spin-up at the L-H transition is expected due to increasing $\left.T_{i}\right|_{\mathrm{pt}}$ and steepening gradients, thus decreasing $L_{\phi}$. The strong dependence on
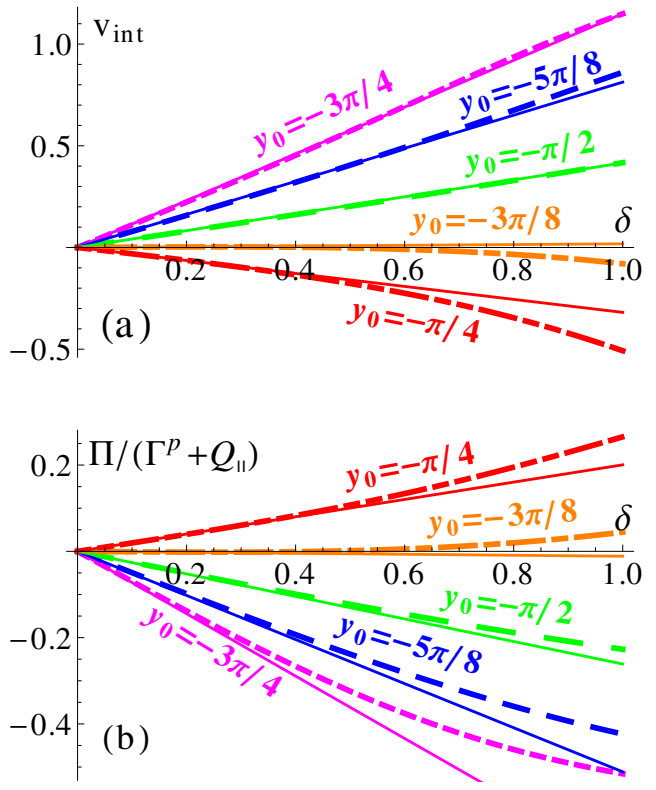

Figure 6. Normalized intrinsic rotation velocity (a) and unbalanced NBI fraction for zero rotation (b), plotted against drift orbit width $\delta$ for several values of the poloidal X-point angle $y_{0}$, with numerical integrals (thick dashed) and analytical linearizations (thin solid).

poloidal X-point angle $y_{0}$ is striking, with a $22^{\circ}$ inboard $\mathrm{X}$-point having nearly double the rotation of a straightdown X-point and a $22^{\circ}$ outboard X-point having essentially zero intrinsic rotation. This prediction is not yet tested.

Recent experiments on DIII-D applied unbalanced NBI heating to zero out the rotation profile. ${ }^{16}$ This case may be addressed by requiring concurrent power and torque balance, ${ }^{111}$

$$
\begin{gathered}
\frac{P_{\mathrm{NBI}}}{f_{\mathrm{NBI}}}=\left.\left.\left.n_{i}\right|_{\mathrm{pt}} T_{i}\right|_{\mathrm{pt}} v_{t i}\right|_{\mathrm{pt}} L_{\phi} L_{\mathrm{tor}} \frac{B_{\theta}}{B_{0}}\left(\Gamma^{p}+Q_{\|}\right) / f_{c}, \\
f_{\mathrm{unb}} R_{0} 2 \frac{P_{\mathrm{NBI}}}{v_{\mathrm{NBI}}}=\left.\left.n_{i}\right|_{\mathrm{pt}} T_{i}\right|_{\mathrm{pt}} L_{\phi} L_{\mathrm{tor}} R_{0} \frac{B_{\theta} B_{\phi}}{B_{0}^{2}}\left(v_{\mathrm{rig}} \Gamma^{p}+\Pi\right),
\end{gathered}
$$

setting $v_{\text {rig }}=0$, and solving for

$$
f_{\mathrm{unb}}=\frac{f_{c}}{2 f_{\mathrm{NBI}}} \frac{B_{\phi}}{B_{0}} \frac{v_{\mathrm{NBI}}}{\left.v_{t i}\right|_{\mathrm{pt}}} \frac{\Pi}{\Gamma^{p}+Q_{\|}},
$$

with $P_{\mathrm{NBI}}$ the total beam power, $f_{\mathrm{unb}} \doteq\left(P_{\mathrm{NBI}}^{\mathrm{co}}-\right.$ $\left.P_{\mathrm{NBI}}^{\mathrm{ctr}}\right) / P_{\mathrm{NBI}}$ the unbalanced beam fraction, $f_{\mathrm{NBI}}$ the fraction of heating by NBI, $f_{c}$ the fraction of heat transported by ions, and $v_{\mathrm{NBI}}$ the beam ion velocity. The ratio $\Pi /\left(\Gamma^{p}+Q_{\|}\right)$is plotted in Fig. 6(b). Since $v_{\mathrm{NBI}} /\left.v_{t i}\right|_{\mathrm{pt}}$ is typically large, $f_{\text {unb }}$ may be a significant fraction of -1 , as observed by Ref. 16 .

Physical understanding is aided by consideration of the inboard and outboard rotation profiles in real space. Using the approximate solutions $f_{i}^{A}$ from Eq. (30) for 

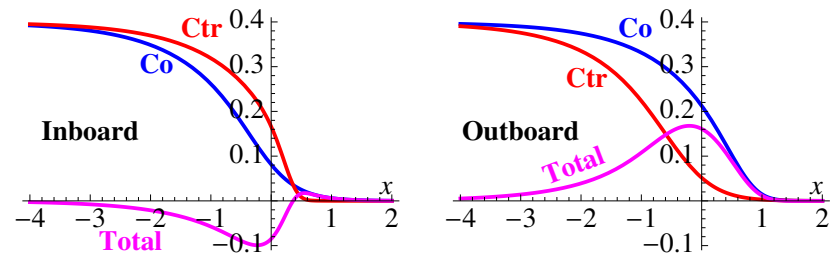

Figure 7. Radial profiles of the toroidal momentum $\int_{-\infty}^{\infty} v f_{i} d v$ (total, purple) at the inboard (left) and outboard (right) midplane, separately showing the absolute contributions of co-going ions $\int_{0}^{\infty}|v| f_{i} d v$ (co, blue) and counter-going ions $\int_{-\infty}^{0}|v| f_{i} d v$ (ctr, red).

$D_{\text {eff }}(v)>2.4$ and $\mathcal{F}_{\bar{y}}\left[f_{i 0}\left(1-\xi^{2} /\left(1+a_{1} D_{\text {eff }}^{1 / 2}+a_{2} D_{\text {eff }}\right)\right)\right]$ for $D_{\text {eff }}(v)<2.4,{ }^{112}$ and inverting the coordinate transforms of Sec. II, one obtains an approximate $f_{i}(x, y, v)$. Fig. 7 shows the radial profiles of toroidal momentum for $v_{\text {rig }}=0$ at the inboard and outboard midplane, in addition to the separate contributions of co- and countergoing ions. The co- and counter-going contributions to the momentum profiles decrease monotonically in radius, showing that the turbulent flux of momentum is always radially outward in this purely diffusive model. There is no pinch or other inward turbulent flux of momentum. However, at the outboard midplane, the steep-gradient region for co-current ions is shifted outward to a region of weaker diffusivity, resulting in a smaller outward flux of co- than counter-current momentum. Although countercurrent ions are shifted outward at the inboard midplane, the diffusivity there is weak so no cancellation results, leaving a net outward flux of counter-current momentum for a nonrotating pedestal top, $v_{\text {rig }}=0$.

Fig. 7 highlights the radially global nature of the spinup, which results from the interaction of nontrivial radial profiles with the geodesic curvature drift and spatial variation of the diffusivity. Recall that $f_{i}$ is the equilibrium distribution function, usually referred to as $F_{0}$ or $F_{M}$ in a radially local model. A standard radially local model neglects radial variation of both $F_{0}$ and its gradient, setting the effect of the orbit shifts on $\nabla F_{0}$ therefore also on the turbulent dynamics - identically to zero. The assumed radial homogeneity of $F_{0}$ and of the magnetic geometry also artificially set the radial gradient of fluctuation intensity identically to zero.

One may also compare with heuristic pictures of edge rotation. For example, ion orbit loss physics appears in the model's outboard co-current and inboard countercurrent rotation layers at the LCFS, because there are less ions on loss orbits $(u>1)$ than confined orbits $(u<1)$. Transport-driven SOL flows, as observed in C-Mod, ${ }^{52}$ also occur naturally in the present model. However, without a radial diffusivity gradient (or some other symmetry-breaking mechanism), neither of these effects drive pedestal-top rotation: $\lim _{\delta, D \rightarrow 0} v_{\text {int }}=0$. To understand this physically, for orbit loss note that the flow layers are caused by the orbit shifts of Fig. 1, then compare co- and counter-going orbit-averaged diffusivities for radially constant diffusivity. For transportdriven flows, take diffusivity as radially constant and consider a poloidal "halfway point" $y_{1 / 2}$ defined such that $\int_{y_{0}}^{y_{1 / 2}} D\left(y^{\prime}\right) d y^{\prime}=\frac{1}{2} \int_{y_{0}}^{y_{0}+2 \pi} D\left(y^{\prime}\right) d y^{\prime}$. From $y_{0}$ to $y_{1 / 2}$, co- and counter-going ions have both diffused equally out into the SOL to drive SOL flows, and they have an equal amount of diffusion remaining to return to the confined edge and drive flows there.

\section{GENERALIZATIONS AND DISCUSSION}

Many simplifications were needed in order to obtain the tractable Eq. (1). In this section, generalizations reintroducing some effects of the $\mathbf{E} \times \mathbf{B}$ and $\nabla B$ drifts are presented. Other generalizations and the implications of omitted terms are qualitatively discussed.

A spatially-constant poloidal "offset"113 $\mathbf{E} \times \mathbf{B} \mathrm{drift}$ $v_{E}$, normalized to $\left.v_{t i}\right|_{\mathrm{pt}} B_{\theta} / B_{0}$ and defined positive for $E_{r}<0$, and a $\nabla B$ drift, evaluated with $\left.v_{\perp}^{2} \rightarrow 2 v_{t i}\right|_{\mathrm{pt}} ^{2}$, may be reintroduced to Eq. (1), resulting in

$$
\begin{array}{r}
\partial_{t} f_{i}+b_{\phi}\left(v+v_{E}\right) \partial_{y} f_{i}-b_{\phi} \delta\left(1+v^{2}\right)(\sin y) \partial_{x} f_{i} \\
-D(y) \partial_{x}\left(e^{-x} \partial_{x} f_{i}\right)=0 .
\end{array}
$$

Following Sec. II but modifying the definitions of $\bar{x}, \bar{y}$, and $D_{y 0}$ with the substitution $\delta v \rightarrow \delta\left(1+v^{2}\right) /\left(v+v_{E}\right)$, transforming $\bar{y} \rightarrow 1-\bar{y}$ for the modified criterion $b_{\phi}\left(v+v_{E}\right)<0$, and setting $D_{\text {eff }} \doteq D_{y 0} /\left|v+v_{E}\right|$, one may transform Eq. (50) to the form of Eq. (8) and the corresponding flux $\Gamma$ to the form of Eq. (11). The results of Secs. III-V thus hold for Eq. (50), using the new definitions and making the substitution $|v| \rightarrow\left|v+v_{E}\right|$ in Eqs. (31), (32), and (40)-(42). Linearization of Eqs. (43) in small $\delta$ and $v_{E}$ with the new definitions produces identical forms for $\Gamma^{p}$ and $Q_{\|}$, while $\Pi$ linearizes to $\Pi \approx 2 \sqrt{\frac{2}{\pi}} \delta\left(\cos y_{0}-\frac{1}{2} d_{c}\right)\left(g_{1}+3 g_{3}-4 g_{5}\right)+$ $\sqrt{\frac{2}{\pi}} v_{E}\left(2 g_{3}-g_{1}\right)$. Without $v_{E}$, the inclusion of the $\nabla B$ drift typically causes a moderate $(\sim 50 \%)$ enhancement of the co-current intrinsic rotation due to larger drift-orbit excursions. For $D_{0} \gtrsim O(1)$, the $v_{E}$ from a negative (positive) $E_{r}$ induces a counter- (co-)current increment to the intrinsic rotation, due to a flattening of the gradients and corresponding flux reduction for ions with poloidal components of $v_{\|}$and $\mathbf{v}_{E}$ in opposition. For $D_{0}$ much smaller than 1 , the non-linearized momentum flux due to $v_{E}$ can change sign, but this occurs mostly due to particles with $v+v_{E} \approx 0$, for which Eq. (50) becomes inaccurate.

Other effects of $E_{r}$, difficult to incorporate into the present framework, may contribute to rotation in experiment. $E_{r}$ shear likely reduces the size of the $v_{E}$ correction just discussed, due to partial cancellation between the pedestal $E_{r}<0$ and SOL $E_{r}>0$. To some extent, effects of $E_{r}$ shear on the turbulence are implicitly included in the present work via the modeling of experimental turbulence parameters with $D_{\text {turb }}$. Although small by this 
paper's orderings, the divergence of the $\mathbf{E} \times \mathbf{B}$ drift can drive poloidally asymmetric toroidal flows that compete with the corresponding curvature-driven toroidal flows for $n_{i} e E_{r} \sim \nabla p_{i}$ and a straight-down X-point in a fluid model. However, in addition to any kinetic enhancements of the curvature-driven flows, a typical inboard X-point doubles the flow-driving effect of curvature but not of $\nabla \cdot \mathbf{v}_{E} \cdot$

Interpretation of the rotation saturation term $v_{\text {rig }} \Gamma^{p}$ in Eq. (44), a diffusive momentum flux resulting from a momentum gradient, is somewhat nuanced. Formally, the present model is core-fuelled and $v_{\text {rig }} \Gamma^{p}$ represents the convective flux of momentum due to density transport in the presence of bulk rotation. Quasineutrality forces real ion density transport to be coupled to electron physics, possibly introducing corrections to the predicted density flux. Also, in experiment most density fuelling occurs in the outer edge or SOL, as could be modeled by adding a source term to the RHS of Eq. (1). Inside the fuelling radius, this reduces the density gradient and increases the velocity gradient without affecting the total momentum gradient, assuming the neutrals have negligible toroidal velocity. So, in an edge-fuelled plasma, the term $v_{\text {rig }} \Gamma^{p}$ may effectively model turbulent viscosity (diffusive momentum transport without net density transport), which is not directly constrained by quasineutrality.

In the Introduction, the spatial decay of potential fluctuations for increasing $r$ was used to argue that $D_{\text {turb }}$ should also drop with increasing $r$. In transport modeling of the SOL, the inferred diffusivity often increases with $r$. However, this diffusivity is based on a comparison of SOL fluxes with SOL gradients, which are dominated by the contribution of ions with energies much lower than $\left.T_{i}\right|_{\mathrm{pt}}$. Such ions enter the SOL predominantly by radial transport, a large and radially-increasing fraction of which is convective transport due to blobs, leading to large inferred diffusivities. In contrast, the high-energy ions that dominate the pedestal momentum flux enter the SOL primarily due to radial drift orbit excursions so, unlike the low-energy ions, their position in the SOL is uncorrelated with the position of the intermittent blobs and they usually hit empty space, supporting the assumption of radially decreasing $D_{\text {turb }}$ in evaluation of the pedestal momentum flux.

A number of other approximations may also be of importance. Although unlikely to directly cause large momentum flux, collisions cause particles to "forget" their orbits. The collision times of the spin-up-dominating suprathermal pedestal-top ions are typically longer than their pedestal-crossing time, suggesting the spin-up mechanism to be relatively weakly affected by collisions. However, the more collisional lower-energy ions contribute to the toroidal viscosity, thus collisions may modify the saturation mechanism. Note also that Eq. (1) [Eq. (50)] replaces trapped particle orbits with narrow [very fat] passing orbits. Since the very fat passing orbits should greatly overestimate these ions' effect, the typically rather modest difference between the rotation results of Eqs. (1) and (50) suggests that inclusion of particle trapping should not qualitatively change the presented results. Other omitted factors such as flux surface shaping, ELMs, and magnetic field errors may also play a role in rotation in experiments. Finally, recall again that this article takes the turbulent transport parametrization as an input, not calculating it self-consistently.

The asymmetric diffusivity sketched in Fig. 1(a), when evaluated for outboard ballooning and radially increasing fluctuations, predicts a net outward flux of co-current momentum leading to a counter-current rotation increment. As an interesting example, should the fluctuation level inside an internal transport barrier (ITB) be lower than the fluctuation level outside, and should the change in fluctuation level occur over a sufficiently short scale length, the physics of Fig. 1(a) suggests that there should be a counter-current rotation increment inside the ITB, as has in fact been observed in experiment. ${ }^{11,114}$ Further investigation is needed to determine if this effect or other core rotation physics is dominant in this case.

\section{SUMMARY}

In a simple transport model for the tokamak pedestal and SOL, the interaction of magnetic drifts with the spatial variation of inhomogeneous but purely diffusive turbulent transport has been demonstrated to cause residual stress and intrinsic rotation of experimentally relevant magnitude (Fig. 6). The physical origin of the spontaneous rotation is an asymmetry of the orbit-averaged diffusivities for co- and counter-going passing ions (Fig. 1). Equivalently, toroidal angular momentum profiles given by the model (Fig. 7) show that the intrinsic rotation results from a shift between the steep-gradient regions for co- and counter-current ions in the presence of a radial diffusivity gradient, a nontrivial profile effect that is identically set to zero in a standard local model. The dimensional prediction for pedestal-top intrinsic rotation is co-current and proportional to $\left.T_{i}\right|_{\mathrm{pt}} / B_{\theta} L_{\phi}[\mathrm{Eq}$. (46)], in agreement with experimental observations. To achieve null pedestal-top rotation, the model predicts that NBI heating must be order-unity unbalanced in the countercurrent direction [Eq. (49)], as observed in experiment. Through its effect on the orbit shifts, an inboard (outboard) angle of the $\mathrm{X}$-point is predicted to strongly drive co- (counter-) current rotation [Fig. (1)(b)].

\section{ACKNOWLEDGMENTS}

Helpful discussions with A. Chankin, G. Hammett, P. Helander, J. Krommes, K. Lackner, O. Maj, R. McDermott, B. Nold, T. Pütterich, C. Rost, P. Schneider, and B. Scott, and an Alexander von Humboldt Foundation research fellowship are gratefully acknowledged. The work leading to this article was funded by the European Atomic Energy Community and is subject to the 
provisions of the Euratom Treaty.

\section{Appendix A: Model derivation}

In this appendix, Eq. (1) will be obtained as a reduction of the ensemble average of Hahm's collisionless, electrostatic gyrokinetic formulation, ${ }^{90}$ treating approximations consecutively: a purely diffusive ansatz for the transport, a radially thin simple-circular geometry motivated by $L_{\perp} / a \sim \epsilon \ll 1$, equilibrium parallel acceleration ordered out by large safety factor $q \sim \epsilon^{-1}$ and aspect ratio $a / R_{0} \sim \epsilon$, integration over the magnetic moment, and finally the assumption of small poloidal $\mathbf{E} \times \mathbf{B}$ Mach number and a specific form for the turbulent diffusivity.

Splitting the total dimensional distribution function $F_{\text {tot }}$ into its ensemble average $F \doteq\left\langle F_{\text {tot }}\right\rangle$ and fluctuation $\tilde{F} \doteq F_{\text {tot }}-F$ and similarly decomposing the gyroaveraged potential $\Phi_{\text {tot }} \rightarrow \Phi+\tilde{\Phi}$ (denoted by Hahm as $\Psi$ ), the ensemble average of Hahm's Eq. (24) may be written as

$$
\begin{array}{r}
\partial_{T}\left(F B^{*}\right)+\nabla \cdot\left(F B^{*} d_{T} \mathbf{X}\right)+\partial_{V \|}\left(F B^{*} d_{T} V_{\|}\right) \\
=-\nabla \cdot \mathbf{b} \times c\langle\tilde{F} \nabla \tilde{\Phi}\rangle+\frac{e}{m_{i}} \partial_{V \|}\left(\mathbf{B}^{*} \cdot\langle\tilde{F} \nabla \tilde{\Phi}\rangle\right), \\
m_{i} d_{T} V_{\|}=-\left(\mathbf{b}+\frac{m_{i} c}{e B^{*}} V_{\|} \mathbf{b} \times \mathbf{b} \cdot \nabla \mathbf{b}\right) \cdot(M \nabla B+e \nabla \Phi), \\
d_{T} \mathbf{X}=V_{\|} \mathbf{b}+\frac{c}{B^{*}} \mathbf{b} \times\left(\frac{1}{e} M \nabla B+\frac{m_{i}}{e} V_{\|}^{2} \mathbf{b} \cdot \nabla \mathbf{b}+\nabla \Phi\right),
\end{array}
$$

with time $T$, gyrocenter position $\mathbf{X}$, magnetic direction $\mathbf{b}$, parallel gyrocenter velocity $V_{\|}$, magnetic moment $M \doteq m_{i} V_{\perp}^{2} / 2 B$, Jacobian $B^{*} \doteq B+\left(m_{i} c / e\right) V_{\|} \mathbf{b} \cdot \nabla \times \mathbf{b}$, and $\mathbf{B}^{*} \doteq B^{*} \mathbf{b}+\left(m_{i} c / e\right) V_{\|} \mathbf{b} \times \mathbf{b} \cdot \nabla \mathbf{b}$. All quantities are evaluated at the gyrocenter position. Axisymmetry implies that all ensemble averages including $F$ and $\Phi$ are independent of the toroidal angle.

Consider first the two fluctuation terms. Taking $\mathbf{b}$. $\nabla \tilde{\Phi} \times \nabla \tilde{F} \sim k_{\perp}^{2} \tilde{\Phi} \tilde{F}, \mathbf{b} \cdot \nabla \tilde{\Phi} \sim \tilde{\Phi} / q R_{0}$, and $\partial_{V \|} \tilde{F} \sim \tilde{F} / v_{t i}$, the second fluctuation term (turbulent parallel acceleration) is small relative to the first (turbulent $\mathbf{E} \times \mathbf{B}$ transport) by the very small factor $1 / k_{\perp}^{2} \rho_{i} q R_{0}$, and will be neglected from now on. For the $\mathbf{E} \times \mathbf{B}$ transport term, since the turbulent autocorrelation times $\sim L_{\perp} / v_{t i}$ are much shorter than the radial transport time $\sim q R_{0} / v_{t i}$, the radial transport process consists of many small uncorrelated steps. The central limit theorem then suggests that a diffusive transport approximation is wellmotivated. Since parallel wavelengths of the fluctuations are also of order $1 / q R_{0}$, the parallel phase advance of an ion in a turbulent autocorrelation time is of order $L_{\perp} / q R_{0} \lll 1$, so it is unlikely that the $\mathbf{E} \times \mathbf{B}$ diffusivity depends significantly on the ions' parallel velocity. Although gyroaveraging suggests that the diffusivity should have some $M$ dependence, this should not play a central role in the toroidal momentum transport, so neglect that for simplicity. Finally, since the radial gradient of $F$ is much steeper than its poloidal gradient and the gradients of the confining magnetic field, take $-\nabla \cdot \mathbf{b} \times c\langle\tilde{F} \nabla \tilde{\Phi}\rangle \rightarrow \partial_{r}\left[D_{\text {turb }} \partial_{r}\left(F B^{*}\right)\right]$ for some turbulent diffusivity $D_{\text {turb }}$, allowed at this point to have arbitrary spatial dependence.

The topology change from closed to open field lines predominantly involves variation of the poloidal magnetic field in the immediate poloidal vicinity of the $\mathrm{X}$-point. Neglect the details of this variation and retain this dependence only as a SOL boundary condition of outgoing ions at a specific poloidal angle, equivalent to an ideal limiter. Note that such a purely outgoing boundary condition is appropriate for the ions that dominate the spinup mechanism discussed in this article, which have relatively large energies, comparable to the pedestal-top ion temperature.

The remaining variation of the confining magnetic field occurs on the $R_{0}$ scale both poloidally and radially. In contrast, $F$ and $\Phi$ should vary radially on the short length scale $L_{\perp}$, but poloidally on a much longer scale $\gtrsim a$. Since the magnetic gradients are crossed with perpendicular gradients of $F$ or $\Phi$, the terms with minorradial gradients of the magnetic field are small in $L_{\perp} / a$. Since the minor-radial width of the domain of interest is also of order $L_{\perp} \ll R_{0}$, one may neglect the minor-radial variation of magnetic quantities altogether.

Neglecting all other details of magnetic shaping, adopt a radially-thin simple-circular geometry. Specifically, with $\theta$ and $\phi$ the simple poloidal and toroidal angles oriented such that $\hat{r} \times \hat{\theta}=\hat{\phi}$, take magnetic field $\mathbf{B}=$ $\left(B_{\theta} b_{\theta} \hat{\theta}+B_{\phi} b_{\phi} \hat{\phi}\right) R_{0} / R$ with constant magnitudes $B_{\phi}, B_{\theta}$, and $R_{0}$ and signs $b_{\phi}$ and $b_{\theta}$, letting $B_{0} \doteq\left(B_{\theta}^{2}+B_{\phi}^{2}\right)^{1 / 2}$. The minor-radial position is then $r=a+X$, with $a$ constant and $X / a \sim L_{\perp} / a \ll 1$. Consistently neglect $X$ in evaluation of $\mathbf{B}$, major-radial position $R \rightarrow R_{0}+a \cos \theta$, and the metric factors $\rightarrow d X, a d \theta$, and $\left(R_{0}+a \cos \theta\right) d \phi$. In this approximation, $B^{*} \rightarrow B, \nabla \times \mathbf{b}=\mathbf{b} \times(\mathbf{b} \cdot \nabla \mathbf{b})=$ $B^{-1} \mathbf{b} \times \nabla B=-\hat{X}(\sin \theta) B_{\phi} b_{\phi} / B_{0} R$, and $\mathbf{b} \cdot \nabla B=$ $(\sin \theta) B_{\theta} b_{\theta} R_{0} / R^{2}$.

Before any further simplifications, it is worthwhile to consider toroidal angular momentum conservation in the reduced geometry. The dominant contribution to the toroidal angular momentum density is that in the parallel flow of ions, $\overline{p_{\phi, \|}}$, with $p_{\phi, \|} \doteq\left(B_{\phi} b_{\phi} / B_{0}\right) m_{i} R V_{\|} F$ and the overbar indicating velocity space integration $\int d V_{\|} \int B d M$. This can now be straightforwardly evaluated as

$$
\begin{aligned}
\partial_{T} \overline{p_{\phi, \|}} & +\nabla \cdot\left(\overline{p_{\phi, \|} d_{T} \mathbf{X}}-\hat{X} D_{\text {turb }} \partial_{X} \overline{p_{\phi, \|}}\right) \\
& -\overline{\left(p_{\phi, \|} / V_{\|}\right) d_{T} V_{\|}}-\overline{p_{\phi, \|} d_{T} \mathbf{X}} \cdot \nabla \ln R=0 .
\end{aligned}
$$

The four terms indicate time variation, momentum flux, parallel acceleration and change of radius. Recalling Eqs. (A1) and the simplified geometry, the parallel acceleration can be seen to result from the mirror force, parallel electric field $E_{\|}$, and parallel energy loss due to curvature drift up a potential gradient. [Considering the characteristics, the latter contribution results 
from a geometric parallel-perpendicular exchange term, $\mathbf{v}_{E} \cdot\left(V_{\|} \mathbf{b} \cdot \nabla \mathbf{b}\right)$, which appears in the second term of $d_{T} V_{\|}=\mathbf{b} \cdot d_{T} \mathbf{v}+\mathbf{v} \cdot d_{T} \mathbf{b}$.] The change of radius follows from parallel free streaming and the $\mathbf{E} \times \mathbf{B}$ drift. The parallel acceleration due to curvature drift along $\nabla \Phi$ and change of radius due to the $\mathbf{E} \times \mathbf{B}$ drift cancel, jointly conserving angular momentum. By quasineutrality and negligible electron mass, the acceleration by $E_{\|}$must represent momentum redistribution within a flux surface via the electrons, basically cold-ion sound wave physics. The mirror force and the change of radius due to parallel flow correspond to the toroidal Lorentz torque density, as is directly seen by evaluating $F(e / c)\left(B_{\theta} b_{\theta} R_{0}\right) d_{T} \mathbf{X} \cdot \hat{X}$. (Of course, it is exactly this balance that leads to conservation of the canonical toroidal angular momentum.) The combination of these terms with the corresponding electron terms would constitute the torque due to the radial gyrocenter current. In order that the true flux-surface averaged radial current vanish, and with it the corresponding torque, this gyrocenter current is balanced by an opposing ion polarization current, showing in essence that the change in parallel toroidal angular momentum is canceled by a corresponding change in the $\mathbf{E} \times \mathbf{B}$ contribution to the toroidal angular momentum, as has been elegantly and quite generally demonstrated. ${ }^{62,63}$

To obtain a simplest-possible conservative model retaining only the ion parallel toroidal angular momentum, the terms constituting momentum exchange via the electrons and with the $\mathbf{E} \times \mathbf{B}$ rotation must be ordered small relative to the radial transport terms. This may be accomplished, while retaining the radial drifts, by taking $q \sim 1 / \epsilon \gg 1$ and $a / R_{0} \sim \epsilon \ll 1$, as may be seen by comparing the surviving terms of $\left(p_{\phi, \|} / V_{\|}\right) d_{T} V_{\|}$ and $p_{\phi, \|} d_{T} \mathbf{X} \cdot \nabla \ln R$ with the corresponding terms of $\nabla \cdot\left(p_{\phi, \|} d_{T} \mathbf{X}\right) \sim p_{\phi, \|} d_{T} X / L_{\perp}$. Physically, the poloidal magnetic field has been ordered small, and with it the toroidal Lorentz torque. Although these orderings are only modestly satisfied for typical experimental parameters, and although they neglect the physics of particle trapping, they lead to a simple, well-behaved, conservative reduction capturing the largest terms in the edge toroidal momentum balance.

The assumptions of large $q$ and small $a / R_{0}$, combined with the orderings $\partial_{V \|} F \sim F / v_{t i}, \partial_{X} F \sim F / L_{\perp}, \partial_{\theta} F \sim$ $F, \partial_{X} \Phi \sim \Phi / L_{\perp}$, and $\partial_{\theta} \Phi \sim \Phi$ now yield

$$
\partial_{T}\left(F B^{*}\right)+d_{T} \mathbf{X} \cdot \nabla\left(F B^{*}\right)=\partial_{X}\left[D_{\text {turb }} \partial_{X}\left(F B^{*}\right)\right],
$$

with $d_{T} \mathbf{X}$ from Eqs. (A1). Both parallel acceleration and the divergence of $d_{T} \mathbf{X}$ have been neglected relative to advection by $d_{T} \mathbf{X}$.

At this point, integrate out the $M$ dependence. Since $\Phi$ is ensemble-averaged, it varies only on the $L_{\perp}$ scale, thus the $M$-dependent gyroaveraging in $\Phi$ is small in $\rho_{i} / L_{\perp}$. Outside of $F$, the only remaining dependence is in the $\nabla B$ drift. Assuming that the rotation drive is not too sensitive to the details of $F$ 's $M$ dependence, approximate this $M$ with $\left.m_{i} v_{t i}\right|_{\text {pt }} ^{2} / B$. Defining $F_{\|} \doteq \int B^{*} F d M$, one then has

$$
\begin{aligned}
& \partial_{T} F_{\|}+d_{T} \mathbf{X}_{\|} \cdot \nabla F_{\|}=\partial_{X}\left(D_{\mathrm{turb}} \partial_{X} F_{\|}\right), \\
& d_{T} \mathbf{X}_{\|} \doteq V_{\|} \mathbf{b}+\frac{c}{B^{*}} \mathbf{b} \times\left(\frac{m_{i}}{e}\left(\left.v_{t i}\right|_{\mathrm{pt}} ^{2}+V_{\|}^{2}\right) \frac{\nabla B}{B}+\nabla \Phi\right) .
\end{aligned}
$$

Poloidal and radial advection by $\mathbf{v}_{E}$ are formally of the same order, $\left(c / B^{*}\right) \mathbf{b} \times \nabla \Phi \cdot \nabla F_{\|} \sim\left(c / B^{*}\right)\left(F_{\|} \Phi / L_{\perp} a\right)$, thus both may be neglected relative to parallel advection when the poloidal $\mathbf{E} \times \mathbf{B}$ velocity is small relative to the poloidal parallel velocity, $c E_{r} / v_{t i} B_{\theta} \ll 1$.

The turbulent diffusivity is assumed separable with exponential decay in the radial direction, $D_{\text {turb }}=$ $D_{Y}(Y) \exp \left(-X / L_{\phi}\right)$, with $Y \doteq a \theta$. The fact that the diffusivity decays in radius is motivated by extensive experimental data ${ }^{67,81-86}$ and determines the sign of the predicted residual stress. The separability and the specific exponential form of the radial decay are of great technical expedience, but are not central to the qualitative results of the calculation.

Explicitly written in $X, Y, \phi$ coordinates, one then has

$$
\begin{aligned}
& \partial_{T} F_{\|}+\frac{B_{\theta} b_{\theta}}{B_{0}} V_{\|} \partial_{Y} F_{\|} \\
&-\frac{B_{\phi} b_{\phi}}{B_{0}} \frac{1}{\Omega_{i} R_{0}}\left(\left.v_{t i}\right|_{\mathrm{pt}} ^{2}+V_{\|}^{2}\right) \sin \left(\frac{Y}{a}\right) \partial_{X} F_{\|} \\
& \\
& \quad=D_{Y} \partial_{X}\left(e^{-X / L_{\phi}} \partial_{X} F_{\|}\right)
\end{aligned}
$$

which, upon neglect of the $\nabla B$ drift, becomes the dimensional form of Eq. (1).

\section{Appendix B: Uniqueness of Green's Function solution}

In this appendix, the uniqueness of the solution of a weak formulation nearly equivalent to Eq. (8), with boundary conditions $f_{i}=f_{i 0}$ for $u=0, f_{i} \rightarrow 0$ for $u \rightarrow \infty$, and $f_{i} \rightarrow f_{\text {init }}(u)$ for $\bar{y} \rightarrow 0$, will be demonstrated. The nature of the approach to the initial conditions and some restrictions on the initial conditions themselves are given precisely in the following proof. ${ }^{115}$

For any specified constant $\bar{y}_{0}>0$, the function $f_{\mathrm{bc}} \dot{=}$ $f_{i 0} \exp \left(-u^{2} / D_{\text {eff }}\left(\bar{y}+\bar{y}_{0}\right)\right)$ solves Eq. (8) and approaches the initial conditions $f_{\mathrm{bc}, 0} \doteq f_{i 0} \exp \left(-u^{2} / D_{\text {eff }} \bar{y}_{0}\right)$ as $\bar{y} \rightarrow$ 0 . The function $\bar{f} \doteq f_{i}-f_{\mathrm{bc}}$ then solves Eq. (8) with the boundary conditions $\bar{f}=0$ for $u=0, \bar{f} \rightarrow 0$ for $u \rightarrow \infty$, and $\bar{f} \rightarrow \bar{f}_{0} \doteq f_{\text {init }}-f_{\mathrm{bc}, 0}$ for $\bar{y} \rightarrow 0$. Define a weighted $L^{2}$ inner product

$$
\langle\eta, \varpi\rangle \doteq \int_{0}^{\infty} \eta \varpi \frac{d u}{u}
$$

with the associated norm $\|\eta\| \doteq\langle\eta, \eta\rangle^{1 / 2}$, in which $\eta$ and $\varpi$ are functions of $u$. Define the solution $\bar{f}$ on all $\bar{y}>0$ and require that $\|\bar{f}\|^{2}+\left\|\partial_{u} \bar{f}\right\|^{2}$ is well-defined. [More precisely, the solution $\bar{f}$ must belong to the Sobolev space 
$H^{1}$ corresponding to the inner product of Eq. (B1).] Assume that the initial conditions $\bar{f}_{0}(u)$ have well-defined norm $\left\|\bar{f}_{0}\right\|^{2}$. (The periodic boundary conditions in the full problem imply this, given that $\|\bar{f}\|^{2}+\left\|\partial_{u} \bar{f}\right\|^{2}$ is welldefined at $\bar{y}=1$.) Require $\bar{f}$ to solve a weak form of Eq. (8),

$$
\partial_{\bar{y}}\langle\eta, \bar{f}\rangle=-\frac{1}{4} D_{\mathrm{eff}}\left\langle\partial_{u} \eta, \partial_{u} \bar{f}\right\rangle,
$$

for all $\bar{y}>0$ and all $\eta$ with well-defined $\|\eta\|^{2}+\left\|\partial_{u} \eta\right\|^{2}$ (in the Sobolev space). [This form may be obtained from Eq. (8) via integration by parts, for which local analysis shows the boundary contribution at zero must vanish for any solution of Eq. (8) that is $C^{2}$ in $u$. The boundary term also vanishes for the weak solution.] Require that $\bar{f}$ approach the initial conditions $\bar{f}_{0}$ in the sense $\lim _{\bar{y} \rightarrow 0}\left\|\bar{f}-\bar{f}_{0}\right\|=0$, equivalent to the requirement $\lim _{\bar{y} \rightarrow 0}\left\|f_{i}-f_{\text {init }}\right\|=0$.

Let $\bar{f}_{1}$ and $\bar{f}_{2}$ both solve Eq. (B2) with the same initial conditions $\bar{f}_{0}$. Their difference $\bar{f}_{d} \doteq \bar{f}_{1}-\bar{f}_{2}$ must also solve Eq. (B2) but with the homogeneous initial conditions, $\lim _{\bar{y} \rightarrow 0}\left\|\bar{f}_{d}\right\|=0$. Since $\bar{f}_{d}$ may be used as the test function in Eq. (B2), we find

$$
\partial_{\bar{y}}\left\|\bar{f}_{d}\right\|^{2}=-\frac{1}{4} D_{\mathrm{eff}}\left\|\partial_{u} \bar{f}_{d}\right\|^{2}
$$

Combined with $\left\|\bar{f}_{d}\right\| \geq 0$ and $\lim _{\bar{y} \rightarrow 0}\left\|\bar{f}_{d}\right\|=0$, this implies $\left\|\bar{f}_{d}\right\|=0$, thus $\bar{f}_{1}$ and $\bar{f}_{2}$ are equal and the weak solution is unique, both in the $L^{2}$ sense. There may therefore be at most one solution of Eq. (B2) that is continuous and has nondivergent norm [as defined by Eq. (B1)] on all $\bar{y}>0$.

\section{Appendix C: Mapping of nonincreasing functions}

The application of the mapping $\mathcal{F}_{\bar{y}}[\psi]$ to a nonincreasing test function $\psi$ that takes values between 0 and $f_{i 0}$ is nonincreasing: One may replace the integral in Eq. (23) with $\int_{0}^{\infty} \psi_{\text {ext }} \partial_{u} G d \xi$, defining the nonincreasing function $\psi_{\text {ext }}$ to equal $\psi$ for $0 \leq \xi \leq 1$ and 0 for $\xi>1$. As we will show below, there is a $\xi_{0} \geq 0$ such that $\partial_{u} G>0$ for $\xi>\xi_{0}$ and $\partial_{u} G<0$ for $0<\xi<\xi_{0}$. We may then use Eqs. (23) and (26) to bound $\partial_{u} \mathcal{F}_{\bar{y}}[\psi]$ from above:

$$
\begin{aligned}
\partial_{u} \mathcal{F}_{\bar{y}}[\psi] \leq-\frac{2 u}{D_{\mathrm{eff}} \bar{y}} f_{i 0} e^{-u^{2} / D_{\mathrm{eff}} \bar{y}} \\
+\psi_{\text {ext }}\left(\xi_{0}\right) \int_{0}^{\xi_{0}} \partial_{u} G d \xi+\psi_{\mathrm{ext}}\left(\xi_{0}\right) \int_{\xi_{0}}^{\infty} \partial_{u} G d \xi \\
\quad=-\frac{2 u}{D_{\mathrm{eff}} \bar{y}} e^{-u^{2} / D_{\mathrm{eff}} \bar{y}}\left[f_{i 0}-\psi_{\mathrm{ext}}\left(\xi_{0}\right)\right]
\end{aligned}
$$

which is nonpositive for nonincreasing $\psi$, as desired.

Consulting Eq. (25), the sign of $\partial_{u} G$ is the same as that of $g_{s}(\xi) \doteq \xi / u-I_{1}\left(2 u \xi / D_{\text {eff }} \bar{y}\right) / I_{0}\left(2 u \xi / D_{\text {eff }} \bar{y}\right)$. The ratio $I_{1}(w) / I_{0}(w)$ ranges from zero at $w=0$ to one for $w \rightarrow \infty$, with positive slope and negative curvature for all $w>0$. The function $g_{s}$ therefore takes the value zero at $\xi=0$, grows large and positive at large $\xi$, and has monotonically increasing slope. If $g_{s}^{\prime}(0) \geq 0$ (which occurs for $u^{2} \leq D_{\text {eff }} \bar{y}$ ), then $g_{s}$, and therefore also $\partial_{u} G$, is positive for all positive $\xi$. If $g_{s}^{\prime}(0)<0$ then $g_{s}$, and therefore also $\partial_{u} G$, is negative for $\xi$ between 0 and some $\xi_{0}>0$ and is positive for $\xi>\xi_{0}$.

\section{Appendix D: Convergence of small- $D_{\text {eff }}$ approximation}

In this appendix, it is demonstrated that the true relation between $f_{i}$ and $\partial_{u} f_{i}$ for $u>1$ lays between that given by Eq. (36) and the leading-order truncation obtained by omitting Eq. (36)'s last term. In addition, these two approximations approach each other as $D_{\text {eff }} \rightarrow 0$, thus they must also approach the true relation. To make the estimate, we will take the true $\partial_{u} f_{i}$ as known and evaluate the error resulting from the approximate expression for $f_{i}$.

First, rewrite the exact SOL relation between $F$ and $\partial_{u} F$ in the convenient form

$$
F=\left[1+\left(\frac{K_{1}\left(z_{S}\right)}{K_{0}\left(z_{S}\right)}-1\right)\right]\left(-\frac{u}{z_{S}} \partial_{u} F\right)
$$

Defining $\hat{f} \doteq-\frac{1}{2}\left(D_{\text {eff }} / \pi\right)^{1 / 2} \int_{0}^{\bar{y}}\left(\bar{y}-y^{\prime}\right)^{-1 / 2} \partial_{u} f_{i} d y^{\prime}$ and $\chi \doteq \mathcal{L}^{-1}\left(K_{1}\left(z_{S}\right) / K_{0}\left(z_{S}\right)-1\right)$, the exact relation between $f_{i}$ and $\partial_{u} f_{i}$ may be compactly written as

$$
f_{i}=\hat{f}+\int_{0}^{\bar{y}} \chi\left(y^{\prime}\right) \hat{f}\left(\bar{y}-y^{\prime}\right) d y^{\prime} .
$$

Since the true $\partial_{u} f_{i}$ is nonpositive (c. f. Sec. III), the true $\hat{f}$ is nonnegative. As we will show, the function $\chi$ is also nonnegative. This shows that the $f_{i}$ resulting from neglecting the last term of Eq. (D1), or equivalently from neglecting the last term of Eq. (36), underestimates the true $f_{i}$. We will also show that $\chi(\bar{y}) \leq\left(D_{\text {eff }} / 16 \pi u^{2} \bar{y}\right)^{1 / 2}$, which implies [using a change of variables to $w \doteq\left(y^{\prime}-\right.$ $\left.\left.y^{\prime \prime}\right) /\left(\bar{y}-y^{\prime \prime}\right)\right]$ that

$$
\begin{aligned}
& \int_{0}^{\bar{y}} \chi\left(\bar{y}-y^{\prime}\right) \hat{f}\left(y^{\prime}\right) d y^{\prime} \\
& \leq-\frac{D_{\mathrm{eff}}}{8 u \pi} \int_{0}^{\bar{y}} \int_{0}^{\bar{y}} \partial_{u} f_{i}\left(y^{\prime \prime}\right) \frac{H\left(y^{\prime}-y^{\prime \prime}\right)}{\sqrt{\bar{y}-y^{\prime}} \sqrt{y^{\prime}-y^{\prime \prime}} d y^{\prime \prime} d y^{\prime}} \\
& =-\frac{D_{\mathrm{eff}}}{8 u \pi}\left(\int_{0}^{\bar{y}} \partial_{u} f_{i}\left(y^{\prime \prime}\right) d y^{\prime \prime}\right)\left(\int_{0}^{1} \frac{d w}{\sqrt{1-w} \sqrt{w}}\right) \\
& =-\frac{D_{\mathrm{eff}}}{8 u} \int_{0}^{\bar{y}} \partial_{u} f_{i}\left(y^{\prime \prime}\right) d y^{\prime \prime}
\end{aligned}
$$

showing that the true $f_{i}$ is bounded from above by the estimate of Eq. (36), as desired. Since the true solution lays between the two estimates, the relative error of either estimate is bounded from above by their relative 
deviation. Since $\left(\bar{y}-y^{\prime}\right)^{-1 / 2} \geq 1$ for $0 \leq y^{\prime} \leq \bar{y} \leq 1$, one may immediately bound this relative deviation with $\left(\pi D_{\text {eff }}\right)^{1 / 2} / 4 u$, vanishing for $D_{\text {eff }} \rightarrow 0$ as desired.

We must now show that $0 \leq \chi \leq\left(D_{\text {eff }} / 16 \pi u^{2} \bar{y}\right)^{1 / 2}$, as assumed. The inverse transform defining $\chi$ is

$$
\chi(\bar{y})=\frac{1}{2 \pi i} \int_{\epsilon-i \infty}^{\epsilon+i \infty} e^{s \bar{y}}\left(\frac{K_{1}\left(z_{S}\right)}{K_{0}\left(z_{S}\right)}-1\right) d s
$$

with $\epsilon$ positive and infinitesimal. Recall that $z_{S} \propto s^{1 / 2}$ has positive real part, thus the integrand is analytic over the complex plane cut along the nonpositive real axis. One may therefore close the integration path for $\chi$ out to $s \rightarrow-\infty$, avoiding the nonpositive real axis. Consulting AS Eqs. 9.6.8-9 and 9.7.2, the contribution of the legs at infinity and the origin vanish, thus letting $w \doteq \mp i z_{S}$ for the leg just above/below the negative real axis one obtains

$$
\chi(\bar{y})=-\frac{D_{\text {eff }}}{4 \pi u^{2}} \int_{0}^{\infty} e^{-D_{\text {eff }} \bar{y} w^{2} / 4 u^{2}}\left[\frac{K_{1}(i w)}{i K_{0}(i w)}-\frac{K_{1}(-i w)}{i K_{0}(-i w)}\right] w d w .
$$

Using AS Eqs. 9.6.32, 9.6.4, 9.1.4, 9.1.40, and 9.1.28, the square-bracketed factor may be more simply rewritten as $\partial_{w} \ln \left(J_{0}^{2}(w)+Y_{0}^{2}(w)\right)$, which is negative definite, thus $\chi$ is positive definite. A simple plot shows that $-\partial_{w} \ln \left(J_{0}^{2}(w)+Y_{0}^{2}(w)\right)$ is bounded from above by its asymptotic value $1 / w$, obtained with AS Eq. 9.2.28, thus we may bound $\chi$ from above with

$$
\chi(\bar{y})<\frac{D_{\mathrm{eff}}}{4 \pi u^{2}} \int_{0}^{\infty} e^{-D_{\mathrm{eff}} \bar{y} w^{2} / 4 u^{2}} \frac{1}{w} w d w=\frac{D_{\mathrm{eff}}^{1 / 2}}{4 u \sqrt{\pi \bar{y}}},
$$

as desired.

\section{Appendix E: Linearized flux moments}

In this Appendix, the small- $\delta$ linearizations of the fluxes in Eq. (43) are derived.

Linearizing the simple-ballooning $D_{\text {eff }}(v)=2 \pi D_{0}[1+$ $\left.\delta v\left(\cos y_{0}-d_{c} / 2\right)+O\left(\delta^{2}\right)\right] /|v|$, one may immediately obtain the linearized $\Gamma^{s}=f_{i 0}|v|\left[\ln \left(1+2 \pi D_{0} / e^{\gamma}|v|\right)+\right.$ $\left.\delta v\left(\cos y_{0}-d_{c} / 2\right) /\left(1+|v| e^{\gamma} / 2 \pi D_{0}\right)+O\left(\delta^{2}\right)\right]$. For an even $f_{i 0}$, the $\delta^{0}\left(\delta^{1}\right)$ term is even (odd) in $v$, thus the even moments are $\int_{-\infty}^{\infty} v^{2 j} \Gamma^{s} d v=2 \int_{0}^{\infty} v^{2 j+1} f_{i 0} \ln (1+$ $\left.2 \pi D_{0} / e^{\gamma} v\right) d v+O\left(\delta^{2}\right)$ and the odd moments (with an integration by parts) are $\int_{-\infty}^{\infty} v^{2 j+1} \Gamma^{s} d v=$ $2 \delta\left(\cos y_{0}-d_{c} / 2\right) \int_{0}^{\infty} \ln \left(1+2 \pi D_{0} / e^{\gamma} v\right) \partial_{v}\left(v^{2 j+4} f_{i 0}\right) d v+$ $O\left(\delta^{2}\right)$. Although the integrals possess exact solutions for Maxwellian $f_{i 0}$, the special functions involved are not easily interpreted. The basic integral $\int_{0}^{\infty} v^{p} e^{-v^{2} / 2} \ln \left(1+2 \pi D_{0} / e^{\gamma} v\right) d v$ may be approximated by Taylor-expanding $\ln \left(1+2 \pi D_{0} / e^{\gamma} v\right)$ about $v=\sqrt{p}$, the maximum of $v^{p} e^{-v^{2} / 2}$. One obtains surprisingly good accuracy by retaining only the constant term $\ln (1+$ $\left.2 \pi D_{0} / e^{\gamma} p^{1 / 2}\right)$, leading directly to the linearized forms of Eq. (43).

\section{REFERENCES}

${ }^{1}$ E. Strait, T. S. Taylor, A. D. Turnbull, J. R. Ferron, L. L. Lao, B. Rice, O. Sauter, S. J. Thompson, and D. Wróblewski, Phys. Rev. Lett. 74, 2483 (1995).

${ }^{2}$ H. Biglari, P. H. Diamond, and P. W. Terry, Phys. Fluids B 2, 1 (1990).

${ }^{3}$ E. J. Doyle, W. A. Houlberg, Y. Kamada, V. Mukhovatov, T. H. Osborne, A. Polevoi, G. Bateman, J. W. Connor, J. G. Cordey, T. Fujita, et al., Nucl. Fusion 47, S18 (2007).

${ }^{4}$ L.-G. Eriksson, T. Hellsten, M. F. F. Nave, J. Brzozowski, K. Holmström, T. Johnson, J. Ongena, K.-D. Zastrow, and JET-EFDA Contributors, Plasma Phys. Controlled Fusion 51, 044008 (2009).

${ }^{5}$ J.-M. Noterdaeme, E. Righi, V. Chan, J. deGrassie, K. Kirov, M. Mantsinen, M. F. F. Nave, D. Testa, K.-D. Zastrow, R. Budny, et al., Nucl. Fusion 43, 274 (2003).

${ }^{6}$ J. S. deGrassie, Plasma Phys. Controlled Fusion 51, 124047 (2009).

${ }^{7}$ J. S. deGrassie, K. H. Burrell, L. R. Baylor, W. Houlberg, and J. Lohr, Phys. Plasmas 11, 4323 (2004).

${ }^{8}$ J. S. deGrassie, J. E. Rice, K. H. Burrell, R. J. Groebner, and W. M. Solomon, Phys. Plasmas 14, 056115 (2007).

${ }^{9}$ L. Porte, S. Coda, S. Alberti, G. Arnoux, P. Blanchard, A. Bortolon, A. Fasoli, T. P. Goodman, Y. Klimanov, Y. Martin, M. Maslov, A. Scarabosio, and H. Weisen, Nucl. Fusion 47, 952 (2007).

${ }^{10}$ J. S. deGrassie, R. J. Groebner, K. H. Burrell, and W. M. Solomon, Nucl. Fusion 49, 085020 (2009).

${ }^{11}$ J. E. Rice, A. C. Ince-Cushman, M. L. Reinke, Y. Podpaly, M. J. Greenwald, B. LaBombard, and E. S. Marmar, Plasma Phys. Controlled Fusion 50, 124042 (2008).

12 J. E. Rice, W. D. Lee, E. S. Marmar, N. P. Basse, P. T. Bonoli, M. J. Greenwald, A. E. Hubbard, J. W. Hughes, I. H. Hutchinson, A. Ince-Cushman, et al., Phys. Plasmas 11, 2427 (2004).

${ }^{13}$ J. E. Rice, W. D. Lee, E. S. Marmar, P. T. Bonoli, R. S. Granetz, M. J. Greenwald, A. E. Hubbard, I. H. Hutchinson, J. H. Irby, Y. Lin, D. Mossessian, J. A. Snipes, S. M. Wolfe, and S. J. Wukitch, Nucl. Fusion 44, 379 (2004).

${ }^{14}$ W. D. Lee, J. E. Rice, E. S. Marmar, M. J. Greenwald, I. H. Hutchinson, and J. A. Snipes, Phys. Rev. Lett. 91, 205003 (2003).

${ }^{15}$ J. E. Rice, J. W. Hughes, P. H. Diamond, Y. Kosuga, Y. A. Podpaly, M. L. Reinke, M. J. Greenwald, Ö. D. Gürcan, T. S. Hahm, A. E. Hubbard, E. S. Marmar, C. J. McDevitt, and D. G. Whyte, Phys. Rev. Lett. 106, 215001 (2011).

${ }^{16}$ W. M. Solomon, K. H. Burrell, J. S. deGrassie, R. Budny, R. J. Groebner, J. E. Kinsey, G. J. Kramer, T. C. Luce, M. A. Makowski, D. Mikkelsen, et al., Plasma Phys. Controlled Fusion 49, B313 (2007).

${ }^{17}$ H. A. Claassen, H. Gerhauser, A. Rogister, and C. Yarim, Phys. Plasmas 7, 3699 (2000).

${ }^{18}$ R. Singh, P. K. Kaw, A. L. Rogister, and V. Tangri, Phys. Plasmas 13, 042505 (2006).

${ }^{19}$ S. K. Wong and V. S. Chan, Phys. Plasmas 14, 112505 (2007).

${ }^{20}$ S. K. Wong and V. S. Chan, Phys. Plasmas 14, 122501 (2007).

${ }^{21}$ U. Daybelge, C. Yarim, and A. Nicolai, Nucl. Fusion 49, 115007 (2009).

${ }^{22}$ A. L. Rogister, J. E. Rice, A. Nicolai, A. Ince-Cushman, S. Gangadhara, and Alcator C-Mod Group, Nucl. Fusion 42, 1144 (2002).

${ }^{23}$ C. S. Chang and S. Ku, Phys. Plasmas 15, 062510 (2008).

${ }^{24}$ J. E. Rice, A. Ince-Cushman, J. S. deGrassie, L.-G. Eriksson, Y. Sakamoto, A. Scarabosio, A. Bortolon, K. H. Burrell, B. P. Duval, C. Fenzi-Bonizec, et al., Nucl. Fusion 47, 1618 (2007).

${ }^{25}$ S. D. Scott, P. H. Diamond, R. J. Fonck, R. J. Goldston, R. B. Howell, K. P. Jaehnig, G. Schilling, E. J. Synakowski, M. C. Zarnstorff, C. E. Bush, et al., Phys. Rev. Lett. 64, 531 (1990).

${ }^{26}$ A. Kallenbach, H. M. Mayer, G. Fussmann, V. Mertens, U. Stroth, O. Vollmer, and the ASDEX Team, Plasma Phys. 
Controlled Fusion 33, 595 (1991).

${ }^{27}$ J. S. deGrassie, D. R. Baker, K. H. Burrell, P. Gohil, C. M. Greenfield, R. J. Groebner, and D. M. Thomas, Nucl. Fusion 43, 142 (2003).

${ }^{28}$ K. C. Shaing, Phys. Rev. Lett. 86, 640 (2001).

${ }^{29}$ B. Coppi, Nucl. Fusion 42, 1 (2002).

${ }^{30}$ C. J. McDevitt, P. H. Diamond, Ö. D. Gürcan, and T. S. Hahm, Phys. Rev. Lett. 103, 205003 (2009).

${ }^{31}$ C. J. McDevitt and P. H. Diamond, Phys. Plasmas 16, 012301 (2009).

${ }^{32}$ T. S. Hahm, P. H. Diamond, O. D. Gurcan, and G. Rewoldt, Phys. Plasmas 14, 072302 (2007).

${ }^{33}$ A. G. Peeters, C. Angioni, and D. Strintzi, Phys. Rev. Lett. 98, 265003 (2007).

${ }^{34}$ A. G. Peeters, D. Strintzi, Y. Camenen, C. Angioni, F. J. Casson, W. A. Hornsby, and A. P. Snodin, Phys. Plasmas 16, 042310 (2009).

${ }^{35}$ A. G. Peeters, C. Angioni, Y. Camenen, F. J. Casson, W. A. Hornsby, A. P. Snodin, and D. Strintzi, Phys. Plasmas 16, 062311 (2009).

${ }^{36}$ E. S. Yoon and T. S. Hahm, Nucl. Fusion 50, 064006 (2010).

${ }^{37}$ R. Singh, R. Ganesh, R. Singh, P. Kaw, and A. Sen, Nucl. Fusion 51, 013002 (2011).

${ }^{38}$ Ö. D. Gürcan, P. H. Diamond, T. S. Hahm, and R. Singh, Phys. Plasmas 14, 042306 (2007).

${ }^{39}$ R. R. Dominguez and G. M. Staebler, Phys. Fluids B 5, 3876 (1993).

${ }^{40}$ Y. Camenen, A. G. Peeters, C. Angioni, F. J. Casson, W. A. Hornsby, A. P. Snodin, and D. Strintzi, Phys. Rev. Lett. 102, 125001 (2009).

${ }^{41}$ N. Kluy, C. Angioni, Y. Camenen, and A. G. Peeters, Phys. Plasmas 16, 122302 (2009).

${ }^{42}$ P. H. Diamond, C. J. McDevitt, Ö. D. Gürcan, T. S. Hahm, and V. Naulin, Phys. Plasmas 15, 012303 (2008)

${ }^{43}$ P. H. Diamond, C. J. McDevitt, Ö. D. Gürcan, T. S. Hahm, W. X. Wang, E. S. Yoon, I. Holod, Z. Lin, V. Naulin, and R. Singh, Nucl. Fusion 49, 045002 (2009).

${ }^{44}$ Ö. D. Gürcan, P. H. Diamond, P. Hennequin, C. J. McDevitt, X. Garbet, and C. Bourdelle, Phys. Plasmas 17, 112309 (2010).

${ }^{45}$ T. E. Stringer, Phys. Rev. Lett. 22, 770 (1969).

${ }^{46}$ A. B. Hassam, T. M. Antonsen, Jr., J. F. Drake, and C. S. Liu, Phys. Rev. Lett. 66, 309 (1991).

${ }^{47}$ H. Wobig and J. Kißlinger, Plasma Phys. Controlled Fusion 37, 893 (1995)

${ }^{48}$ A. G. Peeters, Phys. Plasmas 5, 2399 (1998)

${ }^{49}$ V. A. Rozhansky and I. Y. Senichenkov, Plasma Phys. Controlled Fusion 52, 065003 (2010).

${ }^{50}$ V. Rozhansky and M. Tendler, Phys. Plasmas 1, 2711 (1994).

${ }^{51}$ A. V. Chankin and W. Kerner, Nucl. Fusion 36, 563 (1996).

${ }^{52}$ B. LaBombard, J. E. Rice, A. E. Hubbard, J. W. Hughes, M. Greenwald, J. Irby, Y. Lin, B. Lipschultz, E. S. Marmar, C. S. Pitcher, N. Smick, S. M. Wolfe, S. J. Wukitch, and the Alcotor Group, Nucl. Fusion 44, 1047 (2004).

${ }^{53}$ V. Rozhansky, P. Molchanov, and S. Voskoboynikov, Plasma Phys. Reports 34, 730 (2008)

${ }^{54}$ I. Holod and Z. Lin, Phys. Plasmas 15, 092302 (2008).

${ }^{55}$ R. E. Waltz, G. M. Staebler, J. Candy, and F. L. Hinton, Phys. Plasmas 14, 122507 (2007).

${ }^{56}$ W. X. Wang, T. S. Hahm, S. Ethier, G. Rewoldt, W. W. Lee, W. M. Tang, S. M. Kaye, and P. H. Diamond, Phys. Rev. Lett. 102, 035005 (2009)

${ }^{57}$ W. X. Wang, T. S. Hahm, S. Ethier, L. E. Zakharov, and P. H. Diamond, Phys. Rev. Lett. 106, 085001 (2011).

${ }^{58}$ W. X. Wang, P. H. Diamond, T. S. Hahm, S. Ethier, G. Rewoldt, and W. M. Tang, Phys. Plasmas 17, 072511 (2010).

${ }^{59}$ X. Garbet, Y. Sarazin, P. Ghendrih, S. Benkadda, P. Beyer, C. Figarella, and I. Voitsekhovitch, Phys. Plasmas 9, 3893 (2002).

${ }^{60}$ P. W. Terry, D. A. Baver, and D. R. Hatch, Phys. Plasmas 16 122305 (2009).
${ }^{61}$ F. I. Parra, M. Barnes, and P. J. Catto, Nucl. Fusion 51, 113001 (2011).

${ }^{62}$ B. Scott and J. Smirnov, Phys. Plasmas 17, 112302 (2010).

${ }^{63}$ A. J. Brizard and N. Tronko, Phys. Plasmas 18, 082307 (2011). ${ }^{64}$ B. Scott, Phys. Plasmas 7, 1845 (2000).

${ }^{65}$ B. D. Scott, Plasma Phys. Controlled Fusion 45, A385 (2003).

${ }^{66}$ J. Neuhauser, D. Coster, H. U. Fahrbach, J. C. Fuchs, G. Haas, A. Herrmann, L. Horton, M. Jakobi, A. Kallenbach, M. Laux, et al., Plasma Phys. Controlled Fusion 44, 855 (2002).

${ }^{67}$ B. LaBombard, J. W. Hughes, D. Mossessian, M. Greenwald, B. Lipschultz, J. L. Terry, and the Alcator C-Mod Team, Nucl. Fusion 45, 1658 (2005).

${ }^{68}$ M. N. A. Beurskens, T. H. Osborne, P. A. Schneider, E. Wolfrum, L. Frassinetti, R. Groebner, P. Lomas, I. Nunes, S. Saarelma, R. Scannell, et al., Phys. Plasmas 18, 056120 (2011).

${ }^{69}$ M. Endler, J. Nucl. Mater. 266-269, 84 (1999).

${ }^{70} \mathrm{~J}$. Bleuel, M. Endler, H. Niedermeyer, M. Schubert, H. Thomsen, and the W7-AS Team, New J. Phys. 4, 38 (2002).

${ }^{71} \mathrm{M}$. Wakatani and A. Hasegawa, Phys. Fluids 27, 611 (1984).

${ }^{72}$ B. D. Scott, New J. Phys. 4, 52 (2002).

${ }^{73}$ B. D. Scott, Phys. Plasmas 12, 062314 (2005).

${ }^{74}$ C. S. Chang, S. Ku, P. H. Diamond, Z. Lin, S. Parker, T. S. Hahm, and N. Samatova, Phys. Plasmas 16, 056108 (2009).

${ }^{75}$ For similar order-of-magnitude estimates, compare diffusive and nondiffusive terms in Eq. (7) of Ref. 29, Eq. (5) of Ref. 38, Eq. (15) of Ref. 43, Eq. (9) of Ref. 30, Eqs. (45)-(47) and (59) of Ref. 31, Eq. (1) of Ref. 44 (in which the $\tilde{P}_{k}$ term should be divided by $v_{t i} \rho_{i} P_{i}$ ), and Eqs. (41) and (56) of Ref. 37. As needed, estimate $\omega_{k} \sim k_{\perp} \rho_{s} c_{s} / L_{\perp}, \chi_{\phi} \sim \tilde{v}_{E}^{2} / k_{\perp} \tilde{v}_{E}, k_{\theta} \sim k_{r} \sim k_{\perp}$, $T_{e} \sim T_{i}, \tilde{p} / p \sim e \tilde{\phi} / T_{e}$, and take the edge momentum gradient to be $\left.\left.M_{i}\right|_{\mathrm{pt}} v_{t i}\right|_{\mathrm{pt}} / L_{\perp}$, with $\left.M_{i}\right|_{\mathrm{pt}} \gtrsim 1 / 10$. For Ref. 43, neglect the resonance in the denominator, estimate $\int d \mathbf{k} N \sim$ $\left(T_{e} / m_{i} \omega_{k} k_{\perp}^{2} \rho_{s}^{2}\right)\left(e \tilde{\phi} / T_{e}\right)^{2}$ and $v_{\mathrm{gr}} \sim 2 k_{\perp}^{2} \rho_{s}^{3} c_{s} / L_{\perp}$. For Ref. 31, note that $c_{s} / v_{A}=\left(\beta_{e} / 2\right)^{1 / 2} \ll 1$ in the edge.

${ }^{76}$ A. G. Peeters and C. Angioni, Phys. Plasmas 12, 072515 (2005).

${ }^{77}$ P. C. Stangeby, The Plasma Boundary of Magnetic Fusion Devices (Institute of Physics, 2000).

${ }^{78}$ J. F. Drake, A. B. Hassam, P. N. Guzdar, C. S. Liu, and D. McCarthy, Nucl. Fusion 32, 1657 (1992).

${ }^{79}$ F. L. Hinton and R. D. Hazeltine, Rev. Mod. Phys. 48, 239 (1976).

${ }^{80}$ A. Rogister, Phys. Plasmas 1, 619 (1994).

${ }^{81}$ C. P. Ritz, H. Lin, T. L. Rhodes, and A. J. Wootton, Phys. Rev. Lett. 65, 2543 (1990).

${ }^{82}$ M. Endler, H. Niedermeyer, L. Giannone, E. Holzhauer, A. Rudyi, G. Theimer, N. Tsois, and ASDEX Team, Nucl. Fusion 35, 1307 (1995).

${ }^{83}$ R. A. Moyer, J. W. Cuthbertson, T. E. Evans, G. D. Porter, and J. G. Watkins, J. Nucl. Mater. 241-243, 633 (1997).

${ }^{84}$ R. A. Moyer, R. Lehmer, J. A. Boedo, J. G. Watkins, X. Xu, J. R. Myra, R. Cohen, D. A. D'Ippolito, T. W. Petrie, and M. J. Schaffer, J. Nucl. Mater. 266-269, 1145 (1999).

${ }^{85}$ C. Silva, B. Gonçalves, C. Hidalgo, M. A. Pedrosa, K. Erents, G. Matthews, and R. A. Pitts, Rev. Sci. Instrum. 75, 4314 (2004).

${ }^{86}$ J. Horacek, J. Adamek, H. W. Müller, J. Seidl, A. H. Nielsen, V. Rohde, F. Mehlmann, C. Ionita, E. Havlíčková, and the ASDEX Upgrade Team, Nucl. Fusion 50, 105001 (2010).

${ }^{87}$ Although it is the fluctuating plasma potential that is relevant for the theory, this quantity is seldom measured. ${ }^{86}$ Except for Ref. 86, the cited articles all measure the floating potential, which contains a nonnegligible contribution from $\tilde{T}_{e}$, but might nevertheless have a similar radial envelope to the fluctuating plasma potential.

${ }^{88}$ T. Stoltzfus-Dueck, Phys. Rev. Lett. 108, 065002 (2012).

${ }^{89}$ J. L. Terry, S. J. Zweben, K. Hallatschek, B. LaBombard, R. J. Maqueda, B. Bai, C. J. Boswell, M. Greenwald, D. Kopon, W. M. Nevins, C. S. Pitcher, B. N. Rogers, D. P. Stotler, and X. Q. Xu, Phys. Plasmas 10, 1739 (2003). 
${ }^{90}$ T. S. Hahm, Phys. Fluids 31, 2670 (1988).

${ }^{91}$ T. Pütterich, P. Schneider, and E. Wolfrum(2011), private communication.

${ }^{92}$ P. J. Catto and R. D. Hazeltine, Phys. Plasmas 1, 1882 (1994).

${ }^{93}$ P. Helander and P. J. Catto, Phys. Plasmas 1, 2213 (1994).

${ }^{94}$ D. E. Baldwin, J. G. Cordey, and C. J. H. Watson, Nucl. Fusion 12, 307 (1972).

${ }^{95}$ L. Farnell and W. G. Gibson, J. Comput. Phys. 198, 65 (2004), App. A.1.

${ }^{96}$ Handbook of Mathematical Functions, edited by M. Abramowitz and I. A. Stegun (U.S. Dept. of Commerce, 1972).

${ }^{97}$ F. Oberhettinger and L. Badii, Tables of Laplace Transforms (Springer-Verlag, 1973).

${ }^{98}$ T. H. Stix, Waves in Plasmas (Springer-Verlag, 1992).

${ }^{99} \mathrm{Cut}$ the complex $s$-plane along the negative real axis, so $\sqrt{s}$ always has positive real part. Close the Bromwich integral contour, vertical and just to the right of the imaginary $s$-axis, in the left half of the plane. Since $G(u, \xi, s)$ is analytic inside the contour, and since the legs at infinity and at the origin contribute nothing, the inverse Laplace transform is just -1 times the contribution of the legs along the negative real axis, which may then be written in terms of $w \doteq|\sqrt{s}|$, using AS Eqs. 9.6.3-4, 9.1.35, and 9.1.3-4, as

$G(u, \xi, \bar{y})=\frac{4 u}{D_{\mathrm{eff}}} \int_{0}^{\infty} e^{-w^{2} \bar{y}} J_{1}\left(\frac{2 w u}{D_{\mathrm{eff}}^{1 / 2}}\right) J_{1}\left(\frac{2 w \xi}{D_{\mathrm{eff}}^{1 / 2}}\right) w d w$

which may be evaluated using Eq. (53) of Stix ${ }^{98}$ Ch. 10, yielding Eq. (15).

${ }^{100}$ I. S. Gradshteyn and I. M. Ryzhik, Table of Integrals, Series, and Products, 5th ed. (Academic Press, San Diego, 1994).

${ }^{101}$ The result of Eq. (18) is in fact always continuous, as long as the initial conditions are absolute-value integrable on $[0,1]$.

${ }^{102}$ The solution approaches the initial conditions pointwise $\left[\lim _{\bar{y} \rightarrow 0} f_{i}(u, \bar{y})=f_{i}(u, 0)\right]$ at all $u$, except possibly at $u=$ 0 and/or $u=1$, as can be fairly straightforwardly demonstrated: begin with Eq. (16), add $\left[f_{i}(u, 0)-f_{i}(u, 0)\right] G$ to the integrand, recall Eq. (17), then use the convenient bounds $I_{1}(w)<e^{w} / 4$ and $\int_{w}^{\infty} e^{-t^{2}} d t<e^{-w^{2}} / 2 w$ to show that $\lim _{\bar{y} \rightarrow 0} \int_{0}^{\infty} G(u, \xi, \bar{y})\left[f_{i}(\xi, 0)-f_{i}(u, 0)\right] d \xi=0$. Under certain restrictions on the initial conditions, the solution may also be shown to approach them in an $L^{2}$ sense (c.f. App. B).

${ }^{103}$ This follows directly from the fact that $g_{b}(w) \doteq\left(I_{0}(w)-1\right) / w^{2}$ is nonnegative and nondecreasing for nonnegative $w$, which in turn follows from the facts that $I_{0}(w) \geq 1=I_{0}(0), I_{0}^{\prime}=I_{1}$, $I_{1}(0)=0$, and $I_{1}^{\prime \prime} \geq 0$, since $g_{b}^{\prime}(w)=\left(2 / w^{3}\right)\left[w I_{1}(w) / 2-\right.$ $\left.\left(I_{0}(w)-1\right)\right]=\left(2 / w^{3}\right) \int_{0}^{w}\left[I_{1}(w) w^{\prime} / w-I_{1}\left(w^{\prime}\right)\right] d w^{\prime}$, in which $I_{1}(w) w^{\prime} / w-I_{1}\left(w^{\prime}\right) \geq 0$ because $I_{1}(0)=0, w \geq w^{\prime}$, and $I_{1}$ is convex.

${ }^{104}$ App. B does not prove the nonexistence of additional solutions that do not meet its restrictions on the initial conditions. However, any such solutions would be rather pathological.

${ }^{105}$ The facts that $\left[I_{1}(w) / I_{0}(w)\right]^{\prime}>0$ for $w \geq 0$ and $I_{1}(w) / I_{0}(w)<$ $1-1 / 2 w$ for $w \geq 1$ imply that $\left(D_{\text {eff }} \bar{y}\right)^{1 / 2} G_{I}$ decreases with increasing $\left(u^{2}+\xi^{2}\right) / D_{\text {eff }} \bar{y}$ at any fixed $u / \xi$ for $\left(u^{2}+\xi^{2}\right) / D_{\text {eff }} \bar{y} \geq$ 1. For $\left(u^{2}+\xi^{2}\right) / D_{\text {eff }} \bar{y}<1, u^{2} / D_{\text {eff }} \bar{y}<1$ implies that $\partial_{\xi}\left[\left(D_{\text {eff }} \bar{y}\right)^{1 / 2} G_{I}\right] \leq 0$, thus $\left(D_{\text {eff }} \bar{y}\right)^{1 / 2} G_{I}$ takes its maximum somewhere on $\xi=0,0 \leq u /\left(D_{\text {eff }} \bar{y}\right)^{1 / 2}<1$.

${ }^{106}$ Since the family of continuous functions $\Gamma_{\Delta}(u) \doteq$ $-\frac{1}{2} D_{y 0} u^{-1} \int_{\Delta}^{1} \partial_{u} \mathcal{F}_{\bar{y}}[\psi] d \bar{y}$ (with parameter $0<\Delta<1$ ) approaches the corresponding $\Gamma(u)=-\frac{1}{2} D_{y 0} u^{-1} \int_{0}^{1} \partial_{u} \mathcal{F}_{\bar{y}}[\psi] d \bar{y}$ uniformly in $u \geq u_{0}$ for any $u_{0}>0$ and bounded, absolutevalue-integrable $\psi$, as may be straightforwardly shown using Eq. (28), one may conclude that $\Gamma$ is continuous for all $u>0$, including at $u=1$.

${ }^{107}$ The large- $D_{\text {eff }}$ series for $\Gamma^{A}$ may be obtained using AS Eqs. 5.1.1, 5.1.11, and 9.6.10 along with Eq. 6.611.4 of Ref. 100.

${ }^{108}$ K. B. Oldham and J. Spanier, J. Math. Anal. Appl. 39, 655 (1972).

${ }^{109}$ We are only interested in the solution for $\bar{y} \leq 1$, but it is technically easier to solve on all $\bar{y}>0$ and discard the $\bar{y}>1$ portion.

${ }^{110} \delta$ and $D_{0}$ were obtained for a pure deuterium plasma with $\left.n_{i}\right|_{\mathrm{pt}}=5 \cdot 10^{19} \mathrm{~m}^{-3},\left.T_{i}\right|_{\mathrm{pt}}=600 \mathrm{eV}, B_{0}=2.5 \mathrm{~T}, q=4$, $L_{\phi}=0.02 \mathrm{~m}, R_{0}=1.7 \mathrm{~m}, B_{\theta}=0.21 \mathrm{~T}$, and an ion heat flux of $3 \mathrm{MW} .{ }^{91} D_{0}$ was chosen to match the dimensional heat flux prediction of Eqs. (43) (with $\left.v_{\perp}^{2} \rightarrow 2 v_{t i}\right|_{\mathrm{pt}} ^{2}$ ) to 3MW. Recalling the normalizations and LCFS transport balance, $D_{0}$ is best thought of as determined by the ratio $\left(L_{p i} / L_{\phi}\right)$ at the LCFS, with small $D_{0}$ proportional to $\left(L_{p i} / L_{\phi}\right)^{2}$ and large $D_{0}$ proportional to $\left(L_{p i} / L_{\phi}\right)^{1}$ for $\delta=0$. [For $\delta>0$, nonvanishing orbit shifts prevent $L_{p i}$ from dropping much below $L_{\phi}$ at the separatrix, even for small $D_{0}$.].

${ }^{111}$ The $\Gamma^{p}$ in Eq. (47) appears as an estimate of the transport of perpendicular heat, taking $\left.v_{\perp}^{2} \sim 2 v_{t i}\right|_{\mathrm{pt}} ^{2}$.

${ }^{112}$ The operand in the second form results from the small- $D_{\text {eff }}$ solution by neglecting the $m \neq 0$ modes, which decay rapidly for $u<1$ when $D_{\text {eff }}$ is small.

${ }^{113}$ Eq. (1) does implicitly retain the portion of the $\mathbf{E} \times \mathbf{B}$ drift that contributes to the rigid toroidal rotation $v_{\text {rig }}$, which should correspondingly be subtracted out of the "offset" $\mathbf{E} \times \mathbf{B}$ drift.

${ }^{114}$ J. E. Rice, R. L. Boivin, P. T. Bonoli, J. A. Goetz, R. S. Granetz, M. J. Greenwald, I. H. Hutchinson, E. S. Marmar, G. Schilling, J. A. Snipes, et al., Nucl. Fusion 41, 277 (2001).

${ }^{115}$ The Green's function solution to the full problem $\mathcal{F}_{\bar{y}}[\bar{\psi}]$ and the corresponding initial conditions $f_{i}(u, 0)$ are consistent with all the assumptions made in App. B. For example, one may show that that the Green's function solution approaches the initial conditions in the given $L^{2}$ norm if the initial conditions are bounded by $\left|f_{i}(u, 0)-f_{i 0}\right| \leq M_{u} u^{\alpha}$ and $\left|f_{i}(u, 0)\right| \leq$ $M_{U} u^{-\beta}$ (for some $\left.M_{u}, \alpha, M_{U}, \beta>0\right)$ and are Hölder continuous (with some strictly positive exponent) outside of a finite number of jump discontinuities, criteria which are met by the actual initial conditions. The integral bounds necessary to prove the preceding statements may be completed using Eqs. (15) [implying $G(u, \xi, \bar{y}) \geq 0$ ], (16) (mostly for small $u$ ), (17), (18) (mostly for large u), (23), (27) (for small u), (28) (for larger $u$ ), $I_{0}^{\prime}=I_{1}$, and the inequalities [assuming $u, w \geq 0]\left(b_{1}+b_{2}\right)^{2} \leq 2\left(b_{1}^{2}+b_{2}^{2}\right), e^{-w} \leq 1,1-e^{-w} \leq w$, $0 \leq I_{1}(w)<e^{w} / 4, e^{w} / \sqrt{2 \pi w}, 1 \leq I_{0}(w) \leq e^{w}, \int_{w}^{\infty} e^{-t^{2}} d t \leq$ $e^{-w^{2}} / 2 w, \operatorname{erf}(w) \leq 1,\left(u-w^{2}\right)^{2} \geq u(\sqrt{u}-w)^{2}, \gamma_{a}>$ $0 \Rightarrow|w+\sqrt{u}|^{\gamma_{a}} \leq 2^{\gamma_{a}}\left[|w-\sqrt{u}|^{\gamma_{a}}+|2 \sqrt{u}|^{\gamma_{a}}\right]$ along with $1 \leq \xi \leq u \Rightarrow \xi^{-(\beta+1 / 2)} \leq u^{-(\beta+1 / 2)}+(u-\xi) /(u-1)$ and $u \geq\left(D_{\text {eff }} \bar{y}\right)^{1 / 2}+1 \Rightarrow \int_{0}^{1}\left|\bar{\partial}_{u} G\right| d \xi \leq\left(2 u / D_{\text {eff }} \bar{y}\right) e^{-(u-1)^{2} / D_{\text {eff }} \bar{y}}$ [follows since $I_{1}(w) / I_{0}(w) \geq w /(2+w)$, which implies $\partial_{u} G \leq 0$ for $\left.0 \leq \xi \leq 1, u \geq\left(D_{\text {eff }} \bar{y}\right)^{1 / 2}+1\right]$. 